\title{
SN REFSDAL: CLASSIFICATION AS A LUMINOUS AND BLUE SN 1987A-LIKE TYPE II SUPERNOVA
}

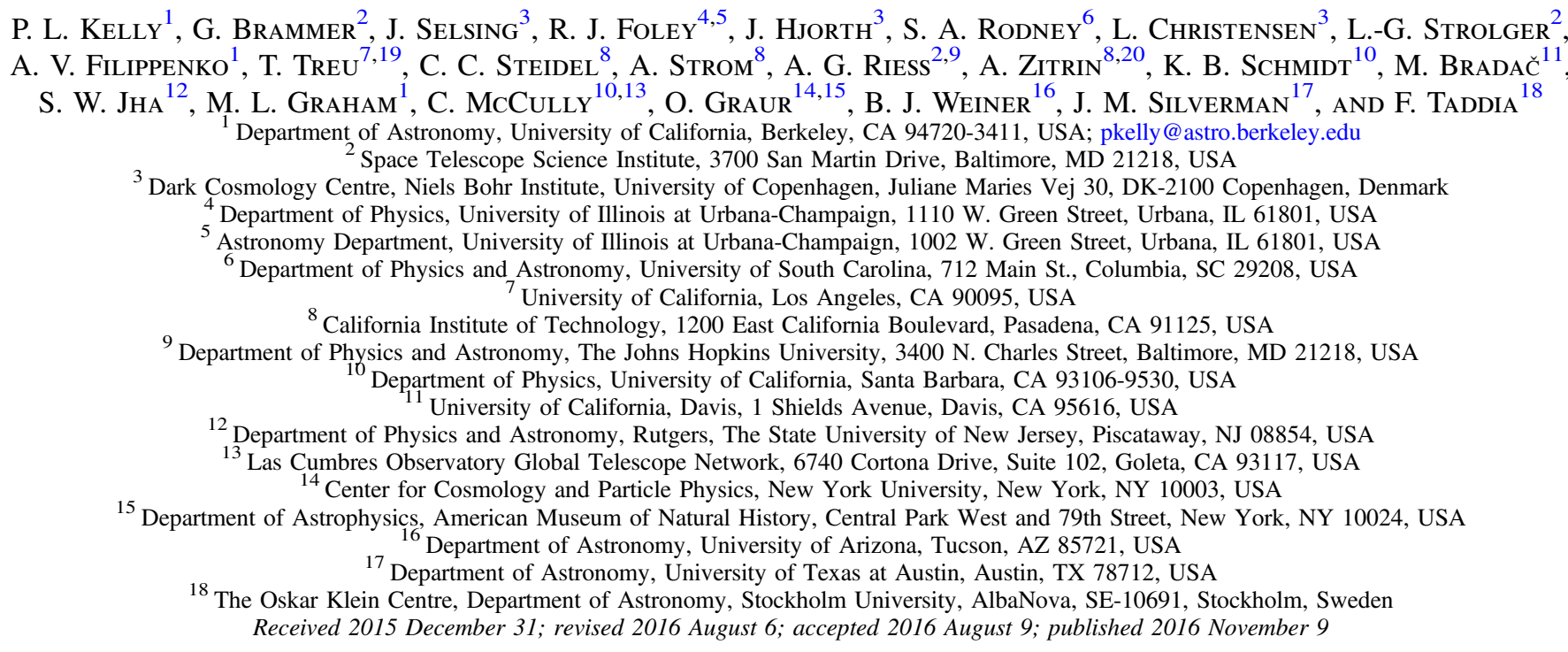

\section{ABSTRACT}

We have acquired Hubble Space Telescope (HST) and Very Large Telescope near-infrared spectra and images of supernova (SN) Refsdal after its discovery as an Einstein cross in fall 2014. The HST light curve of SN Refsdal has a shape consistent with the distinctive, slowly rising light curves of SN 1987A-like SNe, and we find strong evidence for a broad $\mathrm{H} \alpha$ P-Cygni profile and Na I D absorption in the HST grism spectrum at the redshift $(z=1.49)$ of the spiral host galaxy. SNe IIn, largely powered by circumstellar interaction, could provide a good match to the light curve of SN Refsdal, but the spectrum of a SN IIn would not show broad and strong $\mathrm{H} \alpha$ and $\mathrm{NaID}$ absorption. From the grism spectrum, we measure an $\mathrm{H} \alpha$ expansion velocity consistent with those of SN 1987Alike SNe at a similar phase. The luminosity, evolution, and Gaussian profile of the $\mathrm{H} \alpha$ emission of the WFC3 and $\mathrm{X}$-shooter spectra, separated by $\sim 2.5$ months in the rest frame, provide additional evidence that supports the SN 1987A-like classification. In comparison with other examples of SN 1987A-like SNe, photometry of SN Refsdal favors bluer $B-V$ and $V-R$ colors and one of the largest luminosities for the assumed range of potential magnifications. The evolution of the light curve at late times will provide additional evidence about the potential existence of any substantial circumstellar material. Using MOSFIRE and X-shooter spectra, we estimate a subsolar host-galaxy metallicity $(8.3 \pm 0.1 \mathrm{dex}$ and $<8.4 \mathrm{dex}$, respectively) near the explosion site.

Key words: galaxies: clusters: general - galaxies: clusters: individual (MACS J1149+2223) - gravitational lensing: strong - supernovae: general - supernovae: individual (SN Refsdal)

\section{INTRODUCTION}

Refsdal (1964) first considered the possibility that a gravitational lens might create multiple images of a background supernova ( $\mathrm{SN}$ ) explosion. He showed that the time delays between the images of the SN should depend on the distribution of matter in the lens and, geometrically, on the cosmic expansion rate. In Kelly et al. (2015b), we reported the first example of a strongly lensed SN resolved into multiple images, which we found in near-infrared (NIR) Hubble Space Telescope (HST) WFC3 exposures of the MACS J1149+2223 cluster (Ebeling et al. 2001) taken as part of the Grism LensAmplified Survey from Space (GLASS; PI T. Treu; GO-13459; see Schmidt et al. 2014; Treu et al. 2015). The data revealed a total of four images of the SN in an Einstein cross surrounding an early-type galaxy in the cluster. Here we use photometry and

\footnotetext{
19 Packard Fellow.

${ }^{20}$ Hubble Fellow.
}

spectroscopy from the first year after discovery to classify the $\mathrm{SN}$ and characterize its basic properties.

The explosion site of SN Refsdal is close to the tip of a spiral arm of a galaxy at redshift $z=1.49$ (Smith et al. 2009), which is inclined at an angle $i=45 \pm 10^{\circ}$ (Yuan et al. 2011) and is multiply imaged (Zitrin \& Broadhurst 2009) by the potential of the massive MACS J1149+2223 cluster $((1.4 \pm 0.3) \times$ $10^{15} M_{\odot}$; Applegate et al. 2014; Kelly et al. 2014; von der Linden et al. 2014) at $z=0.54$. The cluster lens forms three images of the SN host galaxy that include the explosion site of the SN.

Light that travels toward us on a direct route through the center of the cluster arrives last owing to the greater spatial curvature and gravitational time dilation near the center of the potential (see Treu \& Ellis 2015 for a review). In Kelly et al. (2015b), we predicted that the 2014 appearances were only the next-to-last arrival and that the SN would reappear within several years closer to the center of the cluster. Modeling efforts (Diego et al. 2015; Oguri 2015; Sharon \& Johnson 


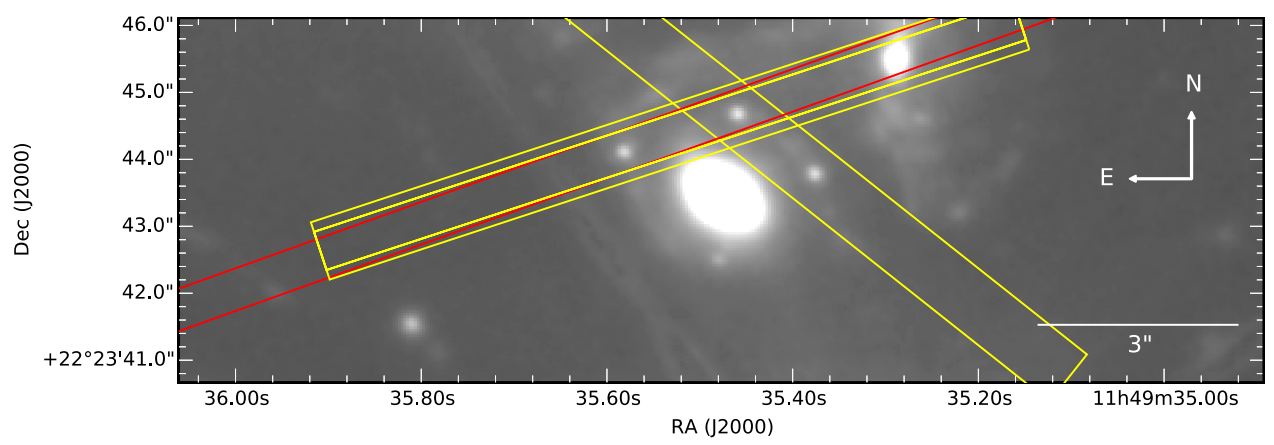

Figure 1. Diagram of the slit positions used for the MOSFIRE (red) and X-shooter (yellow) observations. The image is a coaddition of $F 125 W$ exposures which shows the four SN images forming the Einstein cross.

Table 1

Details of Keck-I MOSFIRE Observations

\begin{tabular}{|c|c|c|c|c|c|c|c|c|c|}
\hline $\begin{array}{l}\text { Date } \\
\text { (MJD) }\end{array}$ & $\begin{array}{l}F 160 W \text { AB } \\
( \pm 0.1 \mathrm{mag})\end{array}$ & $\alpha(\mathrm{J} 2000)$ & $\delta(\mathrm{J} 2000)$ & $\begin{array}{l}\text { Position } \\
\text { Angle }\end{array}$ & $\begin{array}{l}\text { Para. } \\
\text { Angle }\end{array}$ & Airmass & $\begin{array}{l}\text { Total } \\
\text { Exp. (s) }\end{array}$ & $\begin{array}{c}\text { Slit } \\
\text { Width }\end{array}$ & Seeing \\
\hline 56984 & $\sim 25.45(\mathrm{~S} 1) ; \sim 25.5(\mathrm{~S} 2)$ & $11: 49: 35.574$ & $+22: 23: 44.06$ & 109.68 & $82^{\circ}$ & 1.56 & 3578.8 & 0.7 & $0.7^{\mathrm{a}}$ \\
\hline
\end{tabular}

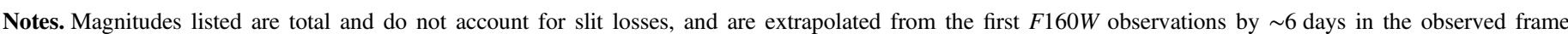
( 2.5 days in the rest frame). Observations were acquired in a sequence of thirty $119.29 \mathrm{~s}$ exposures.

a Determined from the $J$-band image; the FWHM in the $H$ band should be smaller.

2015) sought to make more precise predictions by collecting improved data sets (Grillo et al. 2016; Jauzac et al. 2016; Kawamata et al. 2015; Treu et al. 2016). An imaging campaign with HST (PI P. Kelly; GO-14199) detected the predicted reappearance on December 11, 2015 (UT dates are used throughout this paper; Kelly et al. 2015a), and deep follow-up images will measure the relative time delay with $1 \%-2 \%$ precision.

Either a thermonuclear SN Ia or a core-collapse SN provided a reasonable match to the light curve and colors of SN Refsdal during the first month after discovery, which was made on 2014 November 11. After a $1 \mathrm{hr}$ Keck-I MOSFIRE observation (PI C. Steidel) was not able to detect the SN, an HST Director's Discretionary (DD) time program was carried out from 2014 December 23 through 2015 January 5 (PI P. Kelly; GO-14041) to acquire WFC3 spectra. Instead of fading as would have been expected for a SN Ia, SN Refsdal continued a slow rise in brightness, which made ground-based NIR spectroscopy possible near the peak of the light curve. We obtained Very Large Telescope (VLT) X-shooter spectra through an ESO DD program (PI J. Hjorth; 295.D-5014) in 2015 May and June, approximately six months after discovery. Keck-II DEIMOS observations taken in 2015 December, March, and May yielded no detection of the $\mathrm{SN}$ at optical wavelengths.

Here we show that the spectra and light curve of SN Refsdal are consistent with those of SN 1987A-like SNe, whose prototype was the best-studied SN explosion in recent history. A companion paper (Rodney et al. 2016) presents measurements of the relative time delays and magnifications of the four images in the Einstein cross, and magnitudes measured using point-spread-function (PSF) fitting photometry. In Section 2, we describe the MOSFIRE, DEIMOS, HST grism, and $\mathrm{X}$-shooter spectra that we have collected. The photometric classification of the light curve is discussed in Section 3. Section 4 presents an analysis of the SN spectra, and Section 5 contains measurements of the host-galaxy environment. We summarize the results in Section 6. The methods that we use to process and extract spectra of SN Refsdal, compute
$K$-corrections of photometry of SN Refsdal, and a comparison with the spectra of superluminous SNe (SLSNe) are presented in the Appendix.

\section{DATA}

\subsection{Keck-I MOSFIRE Spectra}

On 2014 November 23, approximately two weeks after discovery, we obtained a $1 \mathrm{hr} H$-band integration with the Multi-Object Spectrometer for Infrared Exploration (MOSFIRE; McLean et al. 2010, 2012) mounted on the $10 \mathrm{~m}$ Keck-I telescope. As we show in Figure 1, a 0"7 wide slit was oriented at position angle (PA) $109^{\circ} .68$ to place the slit across both images $\mathrm{S} 1$ and $\mathrm{S} 2$. The resolving power of the setup was $R=3660$ in the $H$ band $(1.48-1.81 \mu \mathrm{m})$, chosen to be able to have sensitivity to $\mathrm{H} \alpha$ emission at the redshift (1.49) of the face-on spiral galaxy. Table 1 provides an overview of the MOSFIRE observations.

The data were obtained using mask nodding in thirty $119.29 \mathrm{~s}$ exposures, split evenly between integration at positions A and B separated by $12^{\prime \prime}$ along the slit in an alternating sequence, for a total integration of $3578.8 \mathrm{~s}$. The full width at half-maximum (FWHM) intensity of the $J$-band PSF was estimated to be 0.7 during the observations, and the $\mathrm{H}$ band FWHM is expected to have smaller size. The data were reduced using the MOSFIRE Data Reduction Pipeline. ${ }^{21}$

We extract the spectra of images $\mathrm{S} 1$ and S2 using a 4 pixel $(0$ !" 72$)$ width aperture centered on the expected positions of the images of the SN. The locations of S1 and S2 show narrow nebular emission from the host galaxy.

\subsection{Keck-II DEIMOS Data}

We also obtained optical spectra of the field at the $10 \mathrm{~m}$ Keck-II telescope using the DEIMOS spectrograph (Faber et al. 2003). We used multislit masks with $1^{\prime \prime}$ wide slits that

\footnotetext{
${ }^{21}$ https://keck-datareductionpipelines.github.io/MosfireDRP/
} 
Table 2

Seeing During X-shooter Observation Blocks

\begin{tabular}{lcc}
\hline \hline OB & Acquisition Image & Estimated Average \\
& & (FWHM) \\
\hline 1 & 0.69 & 1.00 \\
2 & 0.62 & 0.7 \\
3 & 0.54 & 0.9 \\
\hline
\end{tabular}

Note. To estimate the seeing at the beginning of each $\mathrm{OB}$, two bright stars in the acquisition image are fit with a two-dimensional Gaussian. For all observations a significant worsening of the seeing occurred during the observations. To estimate the average seeing during each OB, we scale the average DIMM seeing by the difference between the measured FWHM in the acquisition images and the DIMM FWHM recorded at the beginning of the OB.

included the positions of SN Refsdal images S1 and S3 on 2014 December 20 with approximately $3 \mathrm{hr}$ of exposure time in fair conditions, and images S2 and S3 on 2015 March 18 (3 hr of exposure in good conditions) and 2015 May 20 (1.5 hr of exposure in fair conditions). We used the 600 line $\mathrm{mm}^{-1}$ grating with a central wavelength of $7200 \AA$, resulting in a wavelength range of 5000-10000 $\AA$ at a scale of $0.65 \AA$ per pixel. A preliminary analysis of the data using a customized version of the DEEP2 pipeline (Cooper et al. 2012; Newman et al. 2013) showed no detectable signal from SN Refsdal over the background.

\subsection{HST G141 Grism Spectra}

As a part of an HST DD program (GO-14041; PI P. Kelly), we obtained thirty orbits of WFC3 G141 grism spectra during the period from 2014 December 23 through 2015 January 5 (13.2 days; 5.3 days in the SN rest frame) when SN Refsdal had $F 160 W \approx 25.1 \mathrm{mag} A B$. During the grism observations, the mean phase of the $\mathrm{SN}$ relative to maximum brightness was $-47 \pm 8$ days. Each $2405.9 \mathrm{~s}$ G141 grism integration was followed by a WFC3 direct imaging $202.9 \mathrm{~s}$ integration through either the $F 125 \mathrm{~W}$ or the $F 160 \mathrm{~W}$ broad-band filters, which are used to align the grism data. The total integration was split equally between observations at telescope orientations of $111^{\circ}$ and $119^{\circ}$, where the spectra of the SN images S2 and S3 were expected to suffer the least contamination from spectra of nearby sources based on our planning simulations and knowledge of the layout of sources in the field.

The WFC3 G141 grism has a resolving power of $\sim 70 \AA$ $\left(\sim 1400 \mathrm{~km} \mathrm{~s}^{-1}\right)$ and a wavelength range of $11000-17000 \AA$ ( $\sim 400-6800 \AA$ in the $z=1.49 \mathrm{SN}$ rest frame). Pointings were made using subpixel offsets to sample the WFC3 PSF completely. The first order of the WFC3 G141 grism has a maximum efficiency of $48 \%$ near $14500 \AA$, while the second order reaches $\sim 8 \%$ at $\sim 11000 \AA$. Each WFC3-IR image has $1024 \times 1024$ pixels covering a 136" $\times 123^{\prime \prime}$ field of view, and the spectra have an average tilt of $\sim 0.5$ relative to the detector's axis.

\subsection{HST Light Curves}

The extraction of the SN light curve from the HST imaging is described in a companion paper (Rodney et al. 2016).

\subsection{VLT X-shooter Spectra}

Data were acquired with the X-shooter echelle spectrograph (Vernet et al. 2011) mounted on Unit Telescope 2 (UT2) of the VLT in three observation blocks (OBs) executed on 2015 May 16 (OB1), June 15 (OB2), and June 16 (OB3). Xshooter covers the entire spectral range $3100-25000 \AA$ by directing incoming light simultaneously to three arms with complementary wavelength coverage. The observations of SN Refsdal were taken in nodding mode where positions A and B were separated by $7^{\prime \prime}$ along the slit.

The fraction of light from a well-centered point source that enters a spectrograph slit depends on the slit width and the FWHM of the PSF. From an $R$-band acquisition image, we can directly estimate the PSF FWHM through X-shooter at the beginning of each OB. The European Southern Observatory (ESO) Ambient Conditions Database ${ }^{22}$ archives an estimate of the seeing at Cerro Paranal from the differential image motion monitor (DIMM). However, the DIMM seeing differs, in general, from the seeing achieved through X-shooter. The DIMM shows that conditions changed significantly during each of the three OBs.

In Table 2, we list estimates for the average seeing during each $\mathrm{OB}$. We measure the seeing through X-shooter at the beginning of the $\mathrm{OB}$ from the FWHM of stars in the acquisition image. We then find the average FWHM recorded by the DIMM during the entire $\mathrm{OB}$, and rescale this average value by the ratio between the DIMM FWHM at the start of the OB and the FWHM measured from the X-shooter acquisition image. The DIMM PSF measured a degradation from a FWHM of $\sim 0$ ". 8 to $\sim 1$ " 6 and then settling at $\sim 1$ " 4 over the course of the OB1 observations. During the OB2 observation, the seeing gradually improved from $\sim 0$ !" 75 to $\sim 0$ " 65 . During the OB3 observation, the seeing evolved from $\sim 0$ ". 7 to $\sim 0$ ". 9 to $\sim 0$ ". 8 .

An overview of the observations is given in Table 3, and we show the slit positions in Figure 1. Observations of SN Refsdal were acquired at a high airmass almost orthogonal to the parallactic angle, so we need to consider carefully the effects of atmospheric dispersion (Filippenko 1982). The telescope tracks the target in images taken at $4700 \AA$, and a tip-tilt mirror corrects for the atmospheric refraction between $4700 \AA$ and the middle of the atmospheric dispersion range for the NIR arm at $13100 \AA$. Since the atmospheric dispersion in the NIR is comparatively small, X-shooter does not have an atmospheric dispersion corrector (ADC) to correct the NIR arm. The relative shift between $13100 \AA$ and $\mathrm{H} \alpha(\sim 16330 \AA)$ is expected to be only $\sim 0$." 1 . The relative atmospheric dispersion across the visible (VIS) arm is greater, and the ADC is not operational. We expect a $\sim 0$ ". 5 shift at [O II] $(\sim 9274 \AA)$ relative to $4700 \AA$, the tracking wavelength where the target is centered on the slit. The slit width in the VIS spectroscopic arm is $0 . " 9$ for OB 1 and 1 !' 2 for $\mathrm{OB} 2$ and OB3. We test that the emission-line ratios in the NIR arm are not significantly affected by comparing spectra from $\mathrm{OB} 1$ and $\mathrm{OB} 2$ to $\mathrm{OB} 3$, for which the PA was closer to the parallactic angle.

\section{PHOTOMETRIC CLASSIFICATION}

During the first three months of observations after discovery on 2014 November 11, SN Refsdal continued to rise in brightness well beyond the time where any normal $\mathrm{SN} \mathrm{Ia,} \mathrm{Ib,} \mathrm{or}$

\footnotetext{
${ }^{22}$ http://archive.eso.org/cms/eso-data/ambient-conditions.html
} 
Table 3

Details of VLT X-shooter Observations

\begin{tabular}{lcccccccc}
\hline \hline OB & $\begin{array}{c}\text { Date } \\
(\mathrm{MJD})\end{array}$ & $\begin{array}{c}F 160 W \mathrm{AB} \\
( \pm 0.1 \mathrm{mag})\end{array}$ & $\alpha(\mathrm{J} 2000)$ & $\delta(\mathrm{J} 2000)$ & $\begin{array}{c}\text { Position } \\
\text { Angle }\end{array}$ & $\begin{array}{c}\text { Para. } \\
\text { Angle }\end{array}$ & $\begin{array}{c}\text { Airmass } \\
\text { Total } \\
\text { Exp. (s) }\end{array}$ & $\begin{array}{c}\text { NIR Slit } \\
\text { Width }\end{array}$ \\
\hline 1 & 57158 & $24.75(\mathrm{~S} 1) ; 24.47(\mathrm{~S} 2)$ & $11: 49: 35.520$ & $+22: 23: 44.44$ & $108^{\circ} .2$ & $175^{\circ}$ & 1.47 & 4800 \\
2 & 57188 & $24.75(\mathrm{~S} 1) ; 24.60(\mathrm{~S} 2)$ & $11: 49: 35.511$ & $+22: 23: 44.65$ & 108.2 & $165^{\circ}$ & 1.51 & 4800 \\
3 & 57189 & $24.60(\mathrm{~S} 2) ; 24.60(\mathrm{~S} 3)$ & $11: 49: 35.406$ & $+22: 23: 44.40$ & 51.6 & $162^{\circ}$ & 1.53 & 4800 \\
\hline
\end{tabular}

Note. Magnitudes listed are total and do not account for slit losses. Observations were acquired in a sequence of four $1200 \mathrm{~s}$ exposures.

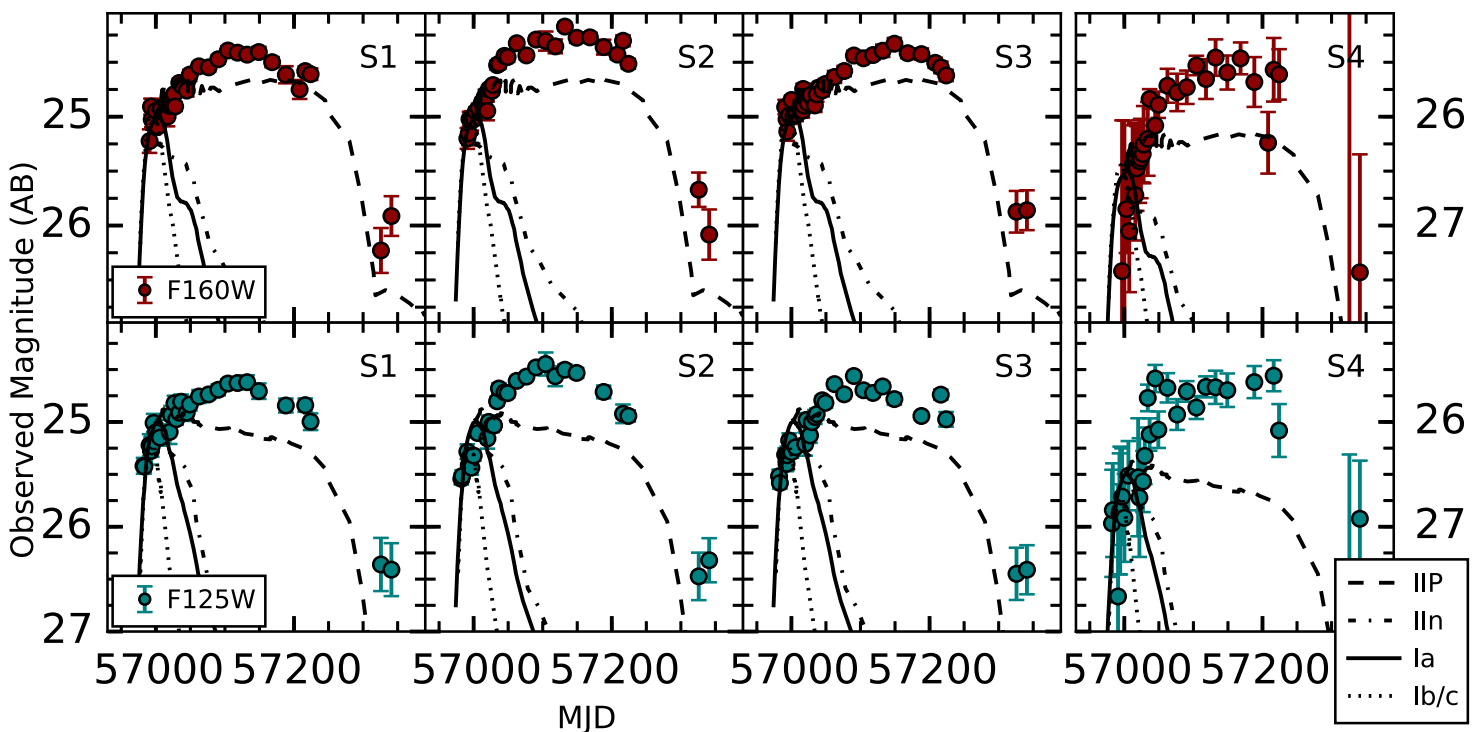

Figure 2. Four columns of panels show light curves of images S1-S4 of SN Refsdal from left to right. (MJD stands for Modified Julian Date.) Top and bottom rows show the $F 160 W$ and $F 125 W$ photometry, respectively. The SN Ia (SN 1994D) and SN Ib/c (SN 1994I) templates are clearly incompatible with the broad light-curve shape of SN Refsdal, while normal SNe IIP ("plateau"; SN 1999em) do not show rising luminosities during their plateau phase. We plot the light curve of SN 1998S, an example of a SN IIn. The presence of strong and broad $\mathrm{H} \alpha$ emission in the spectrum of SN Refsdal shows that it is a SN II (see Figures 6 and 11).

Ic would have reached its peak luminosity (Kelly et al. 2015b). Continued monitoring showed that the light curve rose for $\sim 150$ days, and the SN became similarly incompatible with most normal SN II light curves, which brighten to a "luminosity plateau" over only several days to weeks in the rest frame (Barbon et al. 1979; Doggett \& Branch 1985). In Figure 2, we plot a comparison between the full SN light curves for S1-S4 against the light curves of a typical SN Ia (SN 1994D), SN Ib/c (SN 1994I), SN IIP (SN 1999em), and SN IIn (SN 1998S).

To identify potential matches to the light curve of SN Refsdal, we have performed an extensive search of published SN photometry. While SLSNe have a very small volumetric rate (Quimby et al. 2013), they can exhibit light curves whose peaks are broad (e.g., SN 2008es; SN 2013hx; Miller et al. 2009; Inserra et al. 2016). However, as we show in the Appendix, the equivalent width of $\mathrm{H} \alpha$ emission from SLSNe is substantially too small to match that of SN Refsdal. Furthermore, for the expected magnification listed in Table 4 $(\mu \approx 15)$, SN Refsdal would have an absolute $V$-band magnitude of $-16.9 \pm 0.1$, while SLSNe have absolute magnitudes of $\lesssim-21$. A high extinction $\left(A_{V} \gtrsim 3.5 \mathrm{mag}\right)$ would allow a sufficient luminosity, but the corresponding reddening would require the $\mathrm{SN}$ to be significantly more blue than known SLSNe. Even without lensing magnification, SN Refsdal would have an absolute $V$-band magnitude of only $-19.8 \pm 0.1$.
The slow rise in brightness of SN Refsdal to a broad peak is consistent with the light curve of SN 1987A, a peculiar SN II in the Large Magellanic Cloud (LMC) that is the nearest and brightest SN observed in the last four centuries (Arnett et al. 1989, and references therein). After excluding SLSNe on the basis of their $\mathrm{H} \alpha$ emission, luminosity, and color, only SN 1987A-like SNe as well as a single example of a SN IIn (SN 2005cp) provide approximate matches to the light curve of SN Refsdal.

SN 1987A brightened steadily in the rest-frame optical after initial emission from the shock breakout subsided to reach peak luminosity $~ 84$ days after first light; see Figure 3 of Filippenko (1997) for a comparison with the light curve of a typical SN IIP. The progenitor of SN 1987A was identified as a blue supergiant (Sk $-69^{\circ} 202$; Gilmozzi et al. 1987; Sonneborn et al. $1987)$ in the LMC $(d \approx 50 \mathrm{kpc})$, and more recent $\mathrm{SNe}$ with similar light-curve shapes and spectra are understood to be the result of the explosions of these compact massive stars. Wellstudied examples of nearby SN 1987A-like events include SN 1998A (Pastorello et al. 2005), SN 2000cb and SN 2005ci (Kleiser et al. 2011), SN 2006V and SN 2006au (Taddia et al. 2012), and SN 2009E (Pastorello et al. 2012). These show a wide range of light-curve shapes and colors, explosion energies, and expansion velocities (e.g., Pastorello et al. 2012; Taddia et al. 2012). 
Table 4

Predicted Magnifications

\begin{tabular}{llll}
\hline \hline Model & \multicolumn{1}{c}{$\mu_{\mathrm{S} 1}$} & \multicolumn{1}{c}{$\mu_{\mathrm{S} 2}$} & \multicolumn{1}{c}{$\mu_{\mathrm{S} 3}$} \\
\hline Kelly et al. (2015b) & $\sim 10$ & $\sim 10$ & $\sim 10$ \\
Oguri (2015) & 15.30 & 17.66 & 18.29 \\
Sharon \& Johnson (2015) & $18.5_{-4.5}^{+6.4}$ & $14.4_{-5.5}^{+7.5}$ & $20.5_{-3.9}^{+19.1}$ \\
Grillo et al. (2016) (G12F) & $16.0_{-5.7}^{+1.4}$ & $14.3_{-6.4}^{+4.5}$ & $15.2_{-4.9}^{+4.0}$ \\
Jauzac et al. (2016) & $22.4 \pm 2.0$ & $18.9 \pm 2.3$ & $19.7 \pm 1.7$ \\
Kawamata et al. (2015) & $15.4_{-1.6}^{+1.6}$ & $17.7_{-2.0}^{+2.0}$ & $18.4_{-2.0}^{+2.0}$ \\
\hline
\end{tabular}

Note. Magnifications of images S1-S3 (for which we have spectra) predicted by models of the combined gravitational potential of the early-type galaxy and the MACS1149 galaxy cluster lenses.

In a search of SNe IIn with published photometry, we found a single example, SN SN 2005cp (Kiewe et al. 2012), that shows a light curve similar to that of SN 1987A. SNe IIn are characterized by relatively narrow $\mathrm{H}$ emission lines originating in the interaction between the expanding ejecta and pre-existing circumstellar material (CSM; e.g., Filippenko 1997). The interaction produces narrow Balmer emission and can power a strong underlying continuum.

In Figure 3, we compare the $F 160 \mathrm{~W}$ (rest-frame $\sim R$-band) light curves of the four SN Refsdal images with the $R$-band light curves of SN 1987A-like SNe (PTF 12gcx, SN 2006V, NOOS-005, SN 1987A) and the Type IIn SN 2005cp. The broad-peaked shapes of the light curves of PTF $12 \mathrm{gcx}$ and NOOS-005 provide the best matches to that of SN Refsdal. The PTF 12gcx light curve from Taddia et al. (2016) has dense sampling for 50 days after discovery followed by a 42 day gap in coverage which likely spans the date of maximum brightness. However, the substantial flattening of the light curve before the gap in coverage (see Figure 4 of Taddia et al. 2016) strongly indicates that it has a broad, flat peak similar to that of SN Refsdal.

NOOS-005 was one of the events considered likely to be a SN 1987A-like SN by Pastorello et al. (2012), although its light curve was measured only in the $I$ band and no spectra were taken. Its $I$-band absolute magnitude $\left(M_{I}=-17.51 \mathrm{mag}\right)$ would make it one of the most luminous of SN 1987A-like SNe. For the purpose of computing time delays among the images of SN Refsdal forming the Einstein cross, Rodney et al. (2016) used the light curves of several SN 1987A-like SNe as templates for fitting the multiple light curves of SN Refsdal.

In Figure 4, we show that SN Refsdal has a $F 125 W-F 160 W(\sim V-R$ in the rest frame of the $\mathrm{SN})$ color comparable to those of nearby SN 1987A-like SNe. At an early phase, SN Refsdal has a $F 105 W-F 125 W(\sim B-V)$ color comparable to those of SN 2006V and SN 2006au, the bluest SN 1987A-like SN, but near maximum brightness the color of SN Refsdal is blue in comparison to even SN 2006V and SN 2006au. At almost all epochs and in both colors, SN 2005cp is bluer than SN Refsdal. Here we have applied $K$-corrections to the extinction-corrected colors of the comparison sample (see Table 6). We apply no correction for possible dust extinction to the colors of SN Refsdal, since the low signal-to-noise ratio $(\mathrm{S} / \mathrm{N})$ or low resolution of the spectra do not allow any constraint on absorption by, for example, Na ID or diffuse interstellar bands.

For the sample of SN 1987A-like SNe included in Pastorello et al. (2012), the SNe show a range of absolute magnitudes (in the $V, R$, and $I$ bands) in an approximate range -15 to -17.5 mag. In Figure 5, we show that SN Refsdal may have bluer $B-V$ and $V-R$ colors near maximum light than the comparison sample of SN 1987A-like SNe. The magnifications predicted for sources S1, S2, and S3 are listed in Table 4. From its $M_{B}$ and $M_{V}$ absolute magnitudes, SN Refsdal would be one of the most luminous of the low-redshift SN 1987A-like SNe. For a peak magnitude of $F 160 W \approx 24.2 \mathrm{mag} \mathrm{AB}$ (see Rodney et al. 2016), SN Refsdal would have had $M_{R}=-16.9 \mathrm{mag}$ for an assumed magnification of $\mu=15$.

Taddia et al. (2016) found that PTF 12gcx reached an $r$-band peak on approximately 2012 August 14 . The only photometric constraint on its color is from imaging taken more than three weeks after peak. To estimate the $B-V$ and $V-R$ colors of PTF $12 \mathrm{gcx}$ near peak, we instead average synthetic magnitudes measured from a Palomar $5 \mathrm{~m}$ spectrum taken on 2012 July 26 $(B-V=1.13 \mathrm{mag} ; V-R=0.63 \mathrm{mag})$ and a low-S $/ \mathrm{N}$ Lick $3 \mathrm{~m}$ spectrum taken on 2012 August 23 ( $B-V=0.77 \mathrm{mag}$; $V-R=0.52 \mathrm{mag}$ ). The gri photometry acquired on 2012 September 7 and 8 favor a redder $g-r$ color $(g-r=1.4 \pm 0.3) \quad$ than the synthetic magnitudes $(g-r=0.8-1.1)$, but the $r-i$ colors are in approximate agreement $(r-i \approx 0.3-0.5)$. SN 2006V and SN 2006au were observed in the $r$ band and not in the $R$ band, so we used available spectra as close as possible to maximum light to compute the expected color conversion and find $(V-R)-(V-r) \approx 0.16 \mathrm{mag}$ for both $\mathrm{SNe}$.

\section{SPECTROSCOPIC CLASSIFICATION AND CHARACTERISTICS}

The light curve and colors of SN Refsdal can be matched approximately by those of SN 1987A-like SNe II or, alternatively, SN $2005 \mathrm{cp}$. We next use the WFC 3 grism and the VLT X-shooter spectra to confirm the Type II classification spectroscopically by identifying strong $\mathrm{H} \alpha$ emission. We find compelling evidence for broad and deep absorption by $\mathrm{H} \alpha$ and Na ID, and these features identify SN Refsdal as a SN 1987Alike SN without strong circumstellar interaction.

Figure 6 plots the binned WFC3 grism spectra of SN Refsdal taken in both the $111^{\circ}$ and $119^{\circ}$ telescope orientations at an average phase of $-47 \pm 8$ days relative to maximum light. We plot the weighted average of the raw flux measurements (each $20 \AA$ ) within each $100 \AA$ wavelength bin, and show an uncertainty computed using bootstrapping with replacement. We show, for comparison, a spectrum of SN 1987A obtained at -41 days, of SN 1998A at -40 days (Pastorello et al. 2005), of SN 2006V at -25 days, and of SN 2006au at -19 days (Taddia et al. 2012). These spectra are scaled so that $F 160 W=25.1 \mathrm{mag} \mathrm{AB}$, the average flux of SN Refsdal during the grism observations.

In Figure 7, we plot the wavelength region $8195-10280 \AA$ (5500-6900 $\AA$ in the rest frame) of the WFC3 G141 grism $(-47 \pm 8$ days $)$ and the VLT X-shooter $(+16 \pm 8$ days $)$ spectra. This spectral range shows strong, broad $\mathrm{H} \alpha$ absorption which is present in SN 1987A-like SNe and absent from SNe IIn, including SN 2005cp. Similarly, both the grism and $\mathrm{X}$-shooter spectra show strong, broad NaID absorption features. The spectrum of SN 2005cp (-14 days) plotted for comparison with the grism data contains no similar substantial feature near Na ID. While the post-maximum spectrum of SN $2005 \mathrm{cp}$ (+18 days) plotted for comparison with the VLT spectrum may contain a feature near $\mathrm{Na} I \mathrm{D}$, its minimum is not 

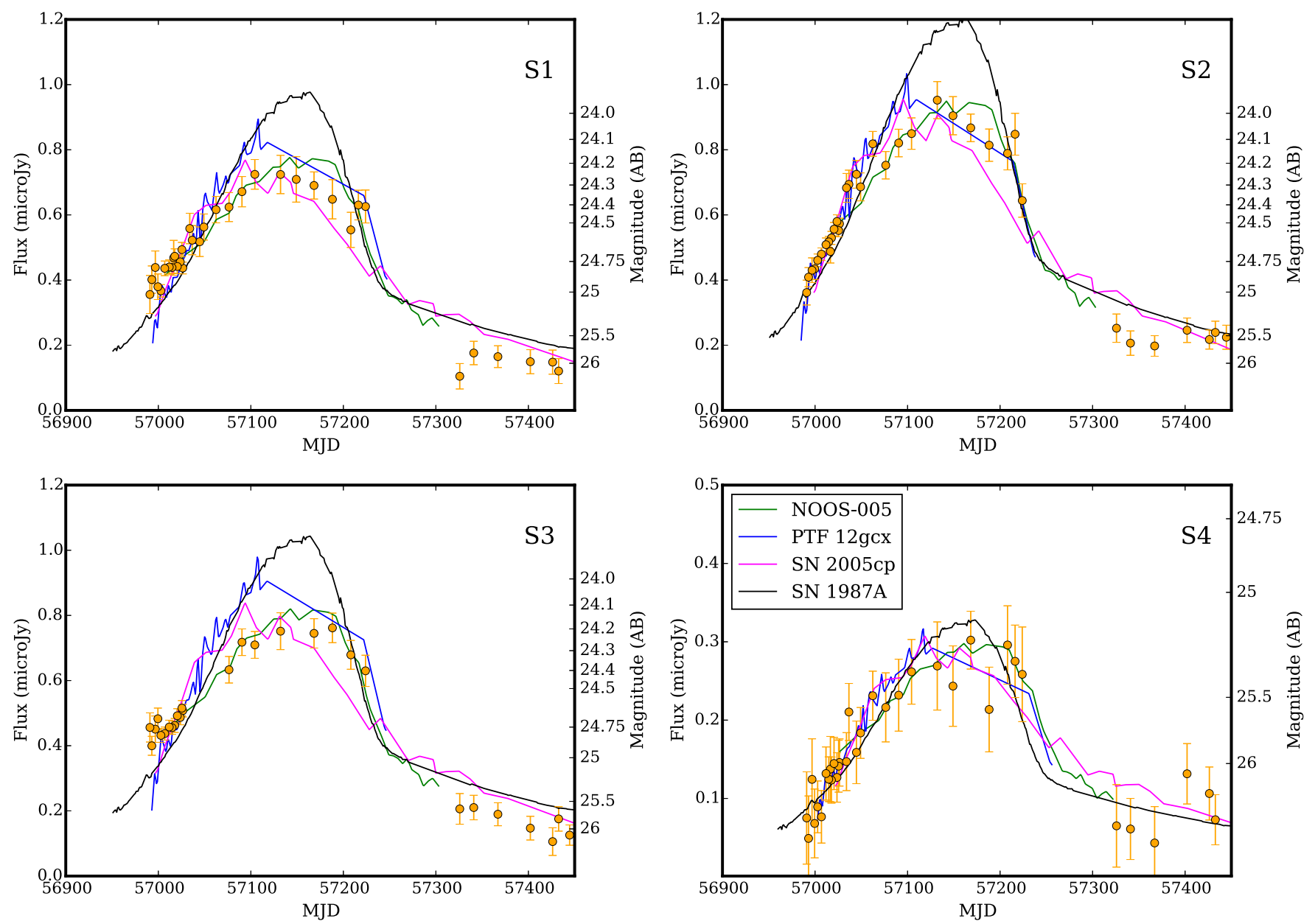

Figure 3. Comparison of SN Refsdal $F 160 \mathrm{~W}$ photometry to the light curves of SN 1987A-like SNe and the Type IIn SN 2005cp. The light curves of PTF $12 \mathrm{gcx}$ and NOOS-005 have the broadest peaks and provide the best matches to that of SN Refsdal among examples of SNe with SN 1987A-like light curves. The light curve of PTF 12gcx contains a gap in coverage during peak, but it exhibits a clear flattening consistent with a broad peak (see Figure 4 of Taddia et al. 2016). The color or spectroscopic properties of NOOS-005 are not known, because it was observed only through the $I$ band, and it reached a luminous absolute magnitude. While SN $2005 \mathrm{cp}$ has a different spectroscopic classification which indicates the presence of significant CSM interaction, it may also be an explosion of a blue supergiant progenitor.

as deep as that present in the spectrum of SN $2006 \mathrm{~V}$ and its shape does not appear to provide a good match to the VLT data.

Figure 8 shows fits of the comparison SN spectra to the WFC3 grism spectrum restricted to respective wavelength regions bracketing $\mathrm{Na} I \mathrm{D}$ and $\mathrm{H} \alpha$. We vary the normalization of each comparison spectrum to find the best $\chi^{2}$ agreement. The Akaike information criterion (AIC; Akaike 1974) yields very strong positive evidence that the SN 1987A-like SNe, which contain prominent $\mathrm{NaID}$ and $\mathrm{H} \alpha$ absorption features, provide a better model than a spectrum of SN 2005cp. A change of 2 in the AIC gives evidence against the model having a greater AIC value, while a difference of 6 constitutes strong evidence (e.g., Kass \& Raftery 1995; Mukherjee et al. 1998).

Despite careful reduction and analysis of the X-shooter spectra, its background contains features we are not able to model. Therefore, we restrict statistical analysis of the $\mathrm{X}$-shooter data to the spectral region containing $\mathrm{H} \alpha$, which is expected to show stronger absorption than Na I D. In Figure 9, we compare the X-shooter data with spectra of SN 1987A-like SNe as well as SN 2005cp at a similar phase and calculate the $\chi^{2}$ agreement within the wavelength range $15000-17000 \AA$ surrounding $\mathrm{H} \alpha$. We use the spectrum of an Sc galaxy redshifted to $z=1.49$ (spiral host galaxy) as a model for the galaxy light, and vary its normalization to find the best match to the data. Using the spectrum of an S0 galaxy at $z=0.54$ (early-type galaxy lens) yields almost identical results. All of the low-redshift comparison spectra are corrected for the Milky Way and host-galaxy extinction values listed in Table 6.

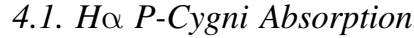

A strong and wide $\mathrm{H} \alpha \mathrm{P}$-Cygni absorption feature is present in the spectra SN 1987A-like SNe II but absent from the spectra of SNe IIn. In SN 1987A-like SNe, the characteristic broad and deep P-Cygni absorption develops as the photosphere recedes into the ejecta. By constrast, the ejecta of SNe IIn collide with CSM and the photosphere generally forms in proximity to the heated, shocked material.

First, we smooth the SN spectrum using a $\sigma=2000 \mathrm{~km} \mathrm{~s}^{-1}$ Gaussian kernel and variance weighting. After removing $>5 \sigma$ outliers from the data, we resmooth the spectrum with outliers removed using the same kernel. We search the smoothed spectrum across the wavelength range $15300-16100 \AA$ to find the absorption minimum. 


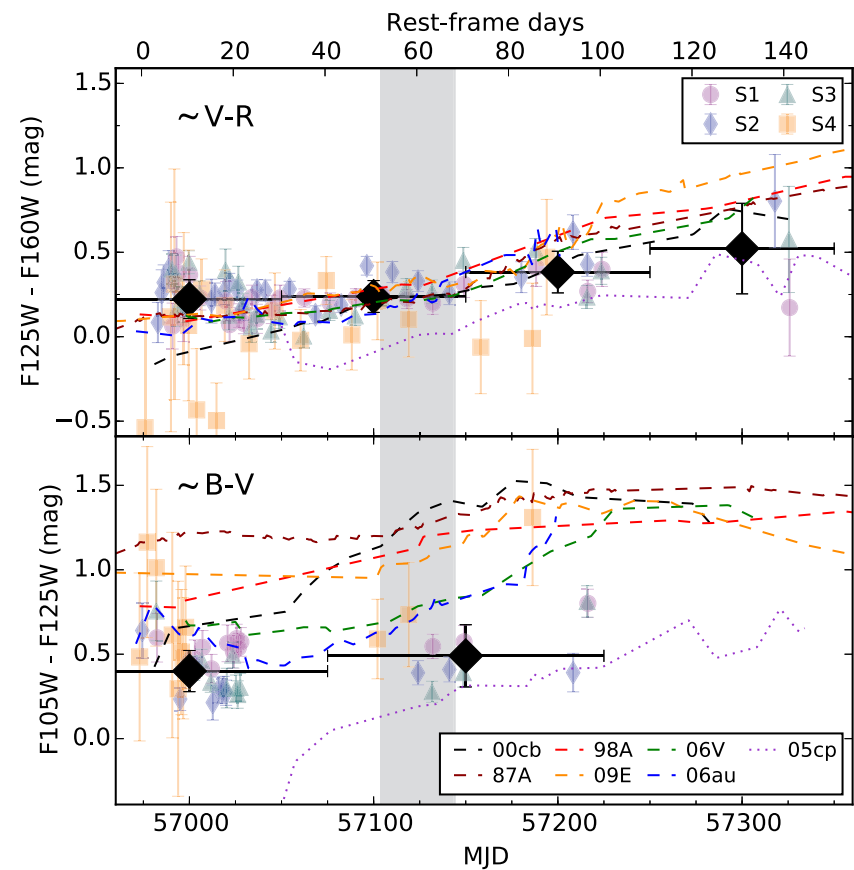

Figure 4. Comparison of the $F 105 W-F 125 W$ and $F 125 W-F 160 W$ colors of SN Refsdal with those of SN 1987A-like SNe as well as the Type In SN $2005 \mathrm{cp}$ as a function of phase relative to maximum light. At all phases for which we have photometry, SN Refsdal shows a $F 125 W-F 160 W(\sim V-R)$ color consistent with those of SN 1987A-like SNe. At an early phase, SN Refsdal exhibits a $F 105 W-F 125 W(\sim B-V)$ color that may be comparable to those of SN 2006V and SN 2006au, the bluest known example of a SN 1987A-like event. Near maximum light, SN Refsdal may be $B-V \approx$ $0.1-0.2$ mag bluer than SN 2006V and SN 2006au. The $B-V$ color of SN $2005 \mathrm{cp}$, a SN IIn whose light curve resembles that of SN 1987A, is bluer than that of SN Refsdal at all phases having photometry.

To determine the uncertainty of the wavelength of the absorption minimum, we use boostrap resampling. For the grism and X-shooter spectra, we assemble the set of all flux measurements at each wavelength taken in both orientations or in all combinations of OBs and SN images, respectively. We resample these sets of measurements with replacement to create the full set of bootstrapped spectra.

The distribution of absorption minima we measure from the bootstrapped WFC3 grism spectra is approximately Gaussian. After rejecting a small population of $>5 \sigma$ outliers, we find an absorption minimum of $-8356 \pm 1105 \mathrm{~km} \mathrm{~s}^{-1}$ $(15880 \pm 60 \AA)$. In contrast, the distribution of absorption minima we measure from the bootstrapped X-shooter spectra has a bimodal shape and is substantially broad, stretching over $\sim 15650-16050 \AA$ A. After removing a small number of outlying measurements close to $15300 \AA$, we constrain the absorption minimum to be $-6465 \pm 2918 \mathrm{~km} \mathrm{~s}^{-1}(15983 \pm 159 \AA)$.

We next perform a data-driven simulation of the WFC3 grism spectrum to determine the statistical significance of finding the absorption feature we identify. As a first step, we smooth the grism spectrum using a $\sigma=2000 \mathrm{~km} \mathrm{~s}^{-1}$ Gaussian kernel, and calculate the residuals of the data from the smoothed spectrum in the wavelength range 14000-16100 $\AA$. Each simulated spectrum is created by replacing the flux at each wavelength in the grism spectrum with a randomly drawn value from the distribution of residuals.

With 10,000 simulated spectra, we compute a test statistic that measures the strength of the absorption relative to the continuum. To estimate the continuum level, we calculate
Table 5

Akaike Information Criteria (AIC)

\begin{tabular}{lrcrrc}
\hline \hline \multirow{2}{*}{ Model } & \multicolumn{2}{c}{$H S T$ WFC3 Grism } & & \multicolumn{2}{c}{ VLT X-shooter } \\
\cline { 2 - 3 } \cline { 5 - 6 } \cline { 5 - 6 } & \multicolumn{1}{c}{$\Delta \mathrm{AIC}$} & $\chi^{2}$ & & $\Delta \mathrm{AIC}$ & $\chi^{2}$ \\
\hline Lorentzian & 0.0 & $197.8\left(N_{\text {param }}=3\right)$ & & 0.0 & $7223.8\left(N_{\text {param }}=5\right)$ \\
Gaussian & -4.7 & $193.0\left(N_{\text {param }}=3\right)$ & & -17.3 & $7206.5\left(N_{\text {param }}=5\right)$ \\
P Cygni & -18.2 & $175.6\left(N_{\text {param }}=5\right)$ & & $\cdots$ & $\cdots$ \\
\hline
\end{tabular}

Note. Increments in the AIC for models of the $\mathrm{H} \alpha$ emission and absorption. A difference greater than 6 is considered strong positive evidence against the model with the higher value. We do not calculate a P-Cygni model for the VLT $\mathrm{X}$-shooter spectrum, because we are only able to constrain the value of the minimum approximately. A Gaussian profile is favored over a Lorentzian profile for both the WFC3 grism and the X-shooter grism data, and a P-Cygni absorption feature is very strongly favored for the WFC3 grism spectrum.

median $\left(f_{1.45-1.55}\right)$, the median flux in the wavelength range 14500-15500 А. We next calculate $\operatorname{median}\left(f_{\text {absorp }}^{\mathrm{H} \alpha}\right)$, the median flux within $\pm 150 \AA$ of the absorption minimum which corresponds to the $2-3 \sigma$ width of SN 1987A-like SN $\mathrm{H} \alpha$ absorption features (see Figure 10). The difference,

$$
\Delta_{\text {absorp }}=\operatorname{median}\left(f_{\text {absorp }}^{\mathrm{H} \alpha}\right)-\operatorname{median}\left(f_{1.45-1.55}\right),
$$

is used as the test statistic to compute a $p$ value. For the grism spectrum, we measure $\Delta_{\text {absorp }}^{\text {grism }}=(-2.5 \pm 0.9) \times 10^{-20}$ $\operatorname{erg~s}^{-1} \mathrm{~cm}^{-2} \AA^{-1}$. We compute the probability of finding a lesser value $\Delta_{\text {absorp }}^{\text {grism }}$ by random chance using the spectra we simulate. We compute $p=0.004$, which provides statistically significant evidence against the hypothesis that the apparent $\mathrm{H} \alpha$ absorption in the grism spectrum is a random artifact.

Given the width of the $\mathrm{H} \alpha$ absorption line and $p=0.004$ significance, we would not expect any other similarly strong absorption feature, and none exists in the range $14000-16100 \AA$. If we instead extend the wavelength range used in the analysis blueward to $11500-15500 \AA$, then the $p$ value increases to $\sim 0.02$. Simulating grism spectra by repeatedly randomly drawing from residuals does not model any covariance in the random noise, although we do not expect a strong covariance.

In the case of the X-shooter spectra, the wavelength of the absorption minimum is poorly constrained, so it is not possible to apply the same statistical test. For the purpose of completeness, however, we calculate the $p$ value for an absorption feature located at $15983 \AA$, the median of the absorption minima measured from the bootstrapped spectra. The continuum is measured as the median of the spectral regions $15300-15600 \AA$ and $16500-17000 \AA$. We calculate $p=0.800$, but note that $15983 \AA$ does not coincide with either peak of the bimodal distribution of minima measured from the bootstrapped spectra.

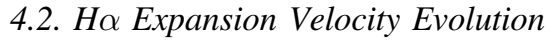

In Figure 11, we plot the constraints on the $\mathrm{H} \alpha$ expansion velocity, and compare the measurements against the expansion velocities of SN 1987A, SN 2006V, and SN 2006au measured by Taddia et al. (2012). The grism measurement at $-47 \pm 8$ days favors an $\mathrm{H} \alpha$ velocity comparable to that of the blue (see Figure 4) SN 1987A-like SN 2006V. The 
Table 6

Nearby Supernovae with SN 1987A-like Light Curves

\begin{tabular}{|c|c|c|c|c|c|c|}
\hline SN & $\begin{array}{c}\text { Host } \\
E(B-V)(\mathrm{mag})\end{array}$ & $\begin{array}{c}\text { Milky Way } \\
E(B-V)(\mathrm{mag})\end{array}$ & $\begin{array}{c}D \\
(\mathrm{Mpc})\end{array}$ & $\begin{array}{l}R \text { - or } r \text {-band } \\
\text { Max. (MJD) }\end{array}$ & $\begin{array}{c}\text { Spectroscopy } \\
\text { Data Set }\end{array}$ & $\begin{array}{c}\text { Photometry } \\
\text { Data Set }\end{array}$ \\
\hline SN 1987A & $0.13(1)$ & $0.06(2)$ & $0.50 \pm 0.005$ & $46933.10 \pm 1.0$ & 3 & 4 \\
\hline SN 1998A & $\sim 0(5)$ & 0.12 & $33 \pm 10$ & $50885.10 \pm 3.9$ & 5 & 6 \\
\hline SN 2006V & $\sim 0$ (7) & 0.029 & $72.7 \pm 5$ & 53824.23 & 9 & 9 \\
\hline SN 2006au & $0.141(8)$ & 0.172 & $46.2 \pm 3.2$ & 53866.25 & 10 & 10 \\
\hline SN 2009E & $0.02(8)$ & 0.02 & $29.97 \pm 2.10(8)$ & $54927.8 \pm 2.8$ & 10 & 10 \\
\hline
\end{tabular}

Note. Publications containing data used for comparison. Many of the spectra were retrieved from WISEREP (http://wiserep.weizmann.ac.il/) (Yaron \& Gal-Yam 2012). Welty et al. (2012) (1); Staveley-Smith et al. (2003) (2); Hamuy \& Suntzeff (1990) (3); Phillips et al. (1988) (http://www.physics.unlv.edu/ jeffery/astro/ sne/spectra/d1980/sn1987a/) (4); Phillips et al. (1990) (ftp://ftp.noao.edu/sn1987a) (5); Pastorello et al. (2005) (6); Kiewe et al. (2012) (7); Silverman et al. (2012) (8); Taddia et al. (2012) (9); Pastorello et al. (2012) (10); Taddia et al. (2016) (11). Distances taken from NASA/IPAC Extragalactic Database (https://ned. ipac.caltech.edu/) except if another citation is provided. Milky Way extinction from Schlafly \& Finkbeiner (2011), except for the case of SN 1987A in the LMC.

approximate $\mathrm{X}$-shooter constraint on the expansion velocity at $+16 \pm 8$ days is consistent with the evolution of the $\mathrm{H} \alpha$ expansion velocity of SN 1987A-like events.

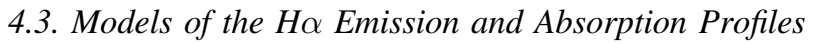

The $\mathrm{H} \alpha$ emission from SNe IIn generally exhibits a Lorentzian profile that arises from Thompson scattering of photons off of free electrons (Chugai 2001; Smith et al. 2010), while Doppler broadening of $\mathrm{H} \alpha$ emission from SN 1987Alike $\mathrm{SNe}$ instead produces approximately Gaussian profiles. Since the line shape contains information about the SN spectroscopic type, we examine which functional form better fits the grism and X-shooter spectra. As shown in Figures 10 and 12, we also model the grism spectrum with a P-Cygni profile including an absorption feature. Our simple model for the P-Cygni absorption consists of two Gaussians, where the difference between their respective centers is set to be equal to twice the sum of their standard deviations.

The model fits provide strong evidence favoring a Gaussian profile over a Lorentzian profile, and very strong evidence for a P-Cygni model for the WFC3 grism spectrum. In Table 5, we list the differences in the AIC to interpret the differences in the $\chi^{2}$ statistics. The AIC penalizes models having a greater number of parameters. A Gaussian model for the absorption feature in the grism spectrum yields a $\sim 4300 \mathrm{~km} \mathrm{~s}^{-1}$ FWHM.

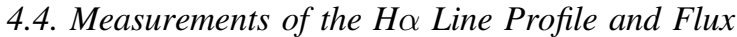

In Figure 13, we compare the total $\mathrm{H} \alpha$ luminosity of SN Refsdal at $-62 \pm 8$ days, $-47 \pm 8$ days, and $+16 \pm 8$ days with the $\mathrm{H} \alpha$ luminosity of SN 1987A-like SNe, as well as SN $2005 \mathrm{cp}$. We measure an upper limit from the MOSFIRE spectrum by fitting for the total flux of a Gaussian profile with $\sigma=900 \mathrm{~km} \mathrm{~s}^{-1}$ for bootstrapped samples using replacement. Before fitting, we remove the background level by fitting it with a linear function. To account for the effect of structure in the background, we shift the center of the Gaussian profile by a random number within $500 \AA$ of the host-galaxy $\mathrm{H} \alpha$ emission. This yields a $3 \sigma$ upper limit of $1.5 \times 10^{-17} \mathrm{erg} \mathrm{s}^{-1} \mathrm{~cm}^{-2}$, which is in approximate agreement with that expected given the MOSFIRE exposure-time calculator. ${ }^{23}$

Using bootstrap resampled spectra and fitting a Gaussian model, we measure $1.1_{-0.6}^{+0.6} \times 10^{-17} \mathrm{erg} \mathrm{s}^{-1} \mathrm{~cm}^{-2}$ and

\footnotetext{
${ }^{23}$ http://www2.keck.hawaii.edu/inst/mosfire/etc.html
}

$2.911_{-0.5}^{+0.5} \times 10^{-17} \mathrm{erg} \mathrm{s}^{-1} \mathrm{~cm}^{-2}$ fluxes from the WFC3 grism and VLT X-shooter spectra, respectively. In these cases, we measure and subtract the background level in adjacent apertures.

The inferred strength and statistically significant change in the $\mathrm{H} \alpha$ emission are consistent with the characteristics of $\mathrm{H} \alpha$ emission from SN 1987A-like SNe. We adopt a fiducial magnification of $\mu=15$ when estimating the $\mathrm{H} \alpha$ luminosity. The plotted gray line shows the systematic shift in the inferred luminosity corresponding to a change in magnification from $\mu=10$ to $\mu=20$ (see Table 4 ).

\subsection{Constraint on the SN Ejecta Mass}

Following Taddia et al. (2012), we scale the parameters of the Blinnikov et al. (2000) model of SN 1987A to estimate the ejecta mass of SN Refsdal. To scale the model, we use the relation $t_{\mathrm{d}} \approx\left(\kappa M_{\mathrm{ej}} / v\right)^{1 / 2}$ from Arnett (1979), where $t_{\mathrm{d}}$ is the diffusion time, $\kappa$ is the mean opacity, and $v$ corresponds to the expansion velocity. We lack direct constraints on the explosion date and the time of the bolometric peak, but SN 1987A-like $\mathrm{SNe}$ exhibit a small dispersion in their rise times; see Table 5 of Pastorello et al. (2012). These assumptions and a scaling according to the $\mathrm{H} \alpha$ expansion velocity yield an estimate for the ejecta mass of $20 \pm 5 M_{\odot}$.

\subsection{Superfit Analysis of the Grism Spectrum}

To identify the spectroscopic classification of a SN, the Superfit (Howell et al. 2005) tool computes the $\chi^{2}$ agreement between an input spectrum and a set of template SN spectra ${ }^{24}$ reddened (or dereddened) by a range of $A_{V}$ values. If the redshift of the $\mathrm{SN}$ is uncertain, template spectra can also be shifted across the possible redshifts of the SN. To calculate the $\chi^{2}$ statistic, Superfit can use the uncertainties of the values in the spectrum.

We apply Superfit to find the SN that best matches the grism spectrum of SN Refsdal. Given the similarity of the light curve of SN Refsdal to those of SN 1987A-like SNe, we add spectra of SN 1998A, SN 2005cp, SN 2006V, SN 2006au, and SN 2009E to the set of Superfit template spectra. Since the position of the lensed source is near the tip of a spiral arm of a lensed galaxy, we fix the $\mathrm{SN}$ redshift to that of the host $(z=1.49)$ to investigate whether we can obtain a satisfactory

\footnotetext{
${ }^{24}$ https://github.com/dahowell/superfit
} 

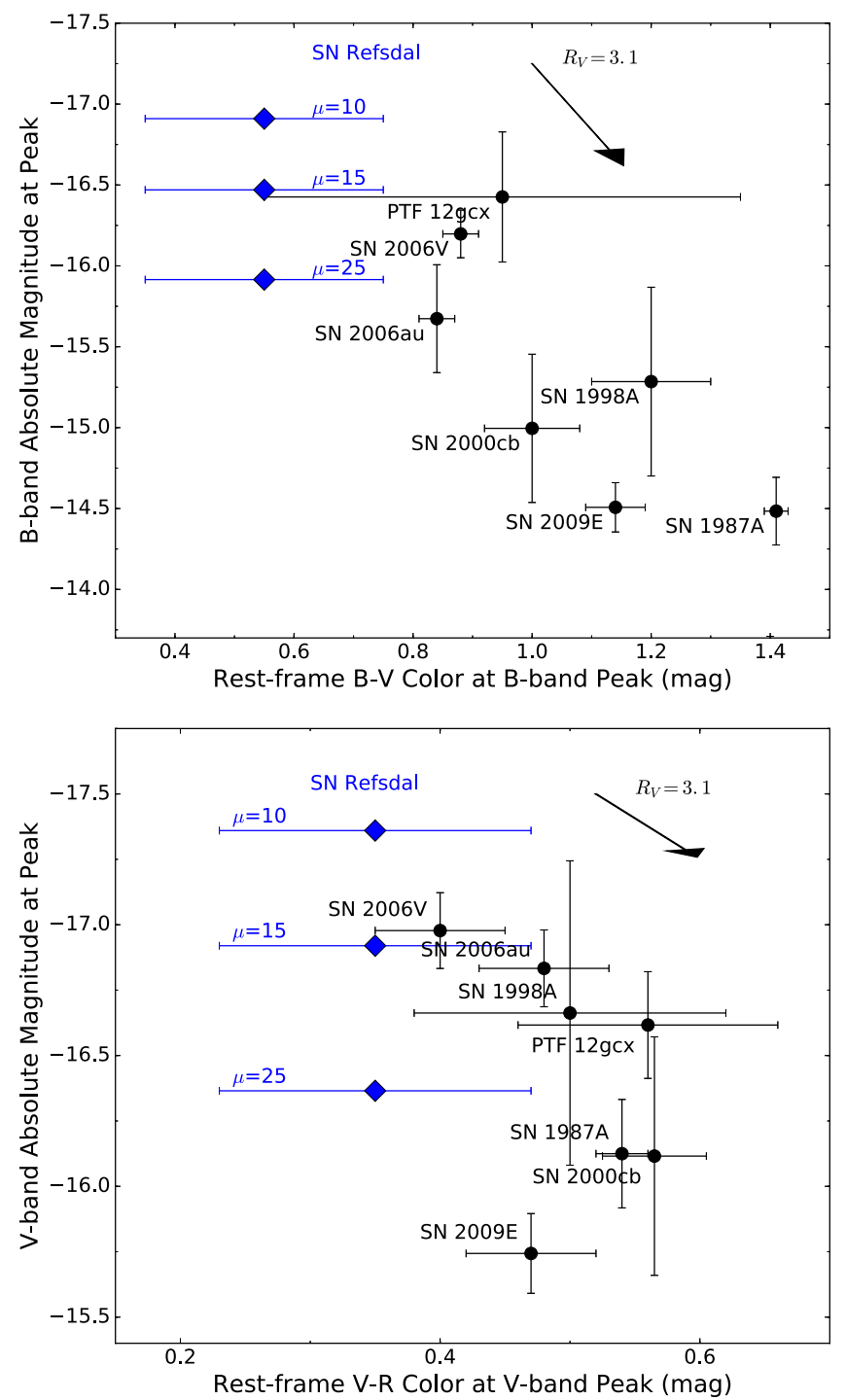

Figure 5. Color and absolute magnitude of SN Refsdal near light-curve peak in comparison with examples of low-redshift SN 1987A-like SNe corrected for host-galaxy and Milky Way extinction. SN Refsdal is likely the most blue (in $B-V$, although not necessarily $V-R$ ) and among the most luminous SN 1987A-like SN near the peak of its light curve. As seen in Figure 3, the slowly evolving light of SN Refsdal may be best matched by those of PTF $12 \mathrm{gcx}$ and NOOS-005. The colors and absolute magnitudes of the SN 1987A-like SNe are corrected for reddening and extinction along the line of sight, but no correction is applied to the photometry of SN Refsdal. For illustration, we plot reddening vectors for $E(B-V)=0.13 \mathrm{mag}$ for an $R_{V}=3.1$ extinction law. Table 4 lists the magnifications of the images of the SN predicted by models of the galaxy and cluster lenses.

fit. We allow the extinction $A_{V}$ to vary between -2 and $+2 \mathrm{mag}$, and we do not include any contribution from the host galaxy since that has already been subtracted. Our input spectrum is not binned and includes the flux measurements taken in both telescope orientations, as well as the uncertainties in the measured fluxes. We adopt five iterations of $5 \sigma$ outlier rejection, which is less aggressive than the default $2.7 \sigma$ rejection.

Table 7 lists the 15 best-fitting template matches to the grism spectrum in the wavelength range 14000-17000 $\AA$. These include only SNe II having $\mathrm{H} \alpha \mathrm{P}-\mathrm{Cygni}$ profiles. If we extend the wavelength range to $11000-17000 \AA$, then a spectrum of SN 2005cp taken $~ 30-50$ days after maximum light provides

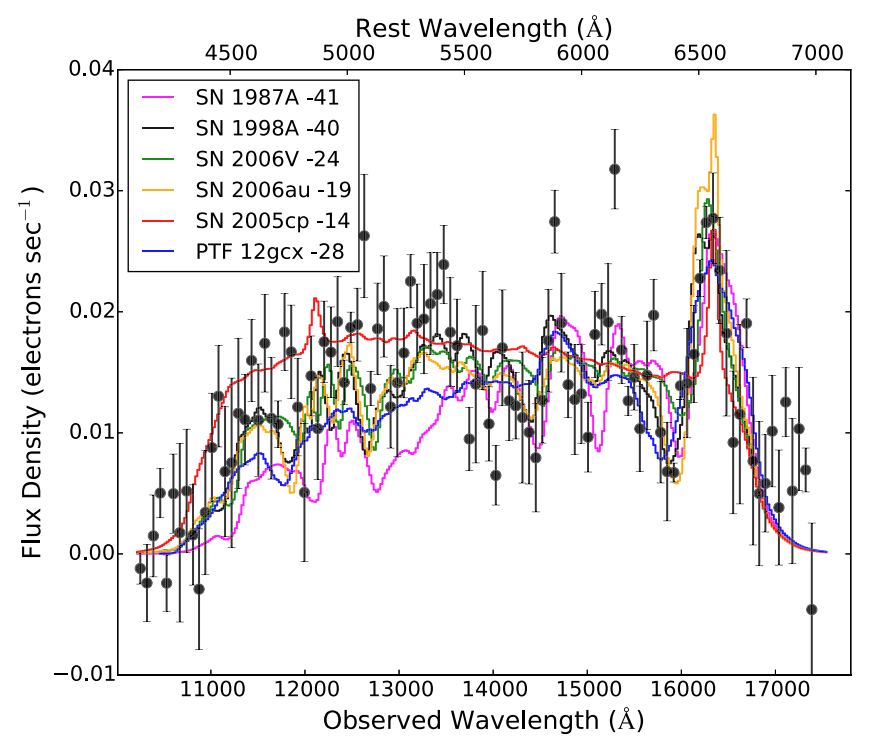

Figure 6. Combination of grism spectra taken in both $111^{\circ}$ and $119^{\circ}$ orientations and binned in wavelength. Each bin is $100 \AA$ in width, and plotted uncertainties are estimated through bootstrapping with replacement fluxes in the $20 \AA$ bins. The grism spectra contributing to this combined spectrum have phases of $-47 \pm 8$ days. SN Refsdal exhibits stronger $\mathrm{H} \alpha$ emission and a bluer continuum at this phase. The color of the SN measured from coadded direct images taken after each grism integration shows agreement with that computed from synthetic magnitudes.

the fifth-best match. All other matches are SNe II having P-Cygni profiles. While the template spectrum of SN 2005cp was taken $\sim 30-50$ days after maximum light, the grism spectra of SN Refsdal were acquired at $-47 \pm 8$ days, and the $\mathrm{H} \alpha$ profile of SN $2005 \mathrm{cp}$ broadened significantly after maximum light as CSM interaction increased (see Figure 9 of Kiewe et al. 2012). As shown in Figure 13, the $\mathrm{H} \alpha$ evolution of SN Refsdal does not appear to be the same as that of SN 2005cp.

\section{THE SN HOST GALAXY AND ENVIRONMENT}

Since the progenitor of SN 1987A was identified as a blue supergiant star (Gilmozzi et al. 1987; Sonneborn et al. 1987), the precursors of SN 1987A-like SNe are believed to be similar - compact, short-lived, massive stars. The properties of the emitting gas near the explosion site of SN Refsdal measured from strong nebular lines should be similar to the properties of the gas that formed the massive progenitor of SN Refsdal. To measure the host-galaxy narrow-line emission, we reduce the spectra in "stare mode" where we do not subtract the off-target spectra. This allows us to avoid subtracting any narrow-line emission from sources in the off-target position which can have bright emission lines. We list the narrow-line measurements in Tables 8 and 9 and the inferred extinction and properties of the ionized gas in Table 10.

As Figure 14 shows, we detect strong nebular emission both near the SN explosion site and from the nuclear region of the host galaxy. The emission from the nuclear region has a significantly broader profile, which we attribute to the rotational motion of the gas. From the best-fit line centers, we measure redshifts of $1.48831 \pm 0.00007$ from the spectrum extracted around the SN position and $1.48837 \pm 0.00007$ for the nuclear region, where the uncertainties include contributions from both line fitting and the wavelength solution. 

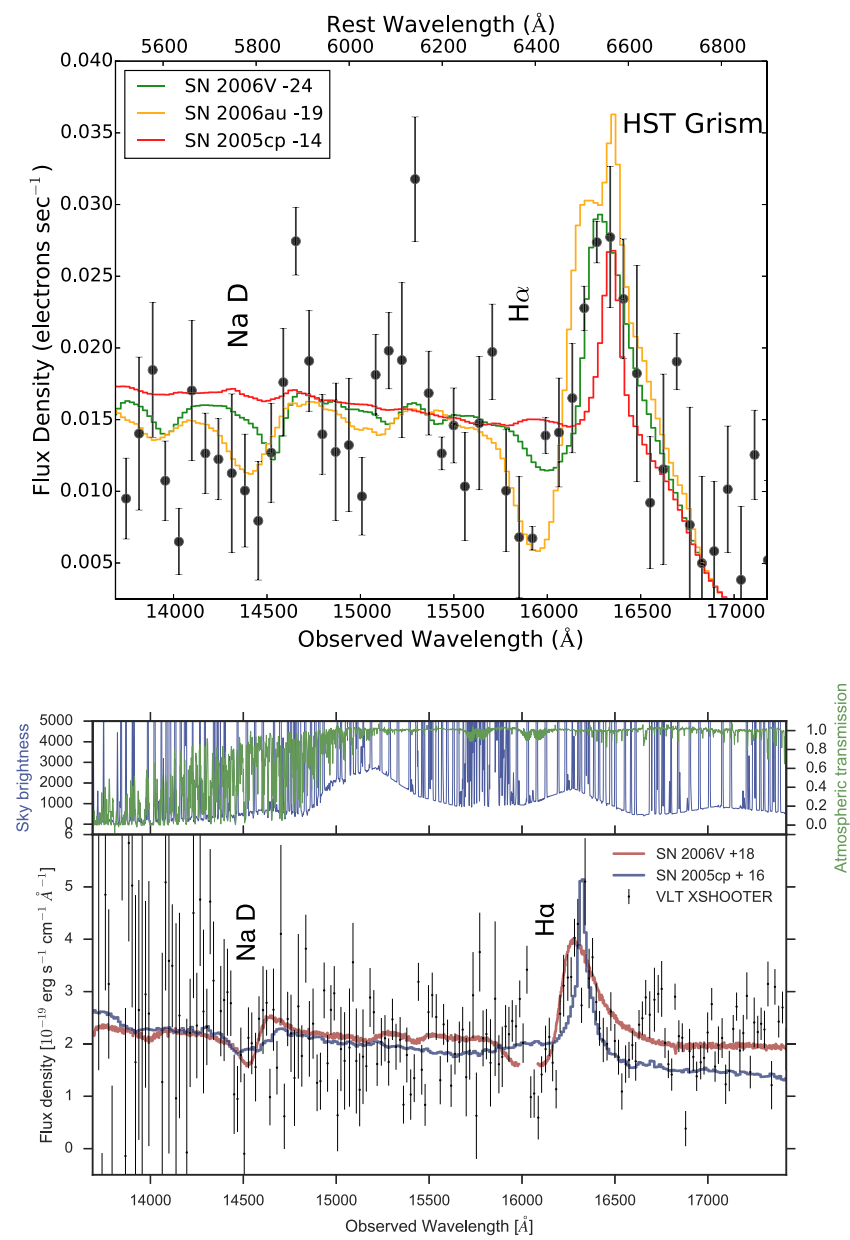

Figure 7. WFC3 G141 grism (upper panel; $-47 \pm 8$ days) and the VLT $\mathrm{X}$-shooter (lower panel; $+16 \pm 8$ days) spectra showing the expected location of $\mathrm{Na} \mathrm{I} \mathrm{D} \mathrm{and} \mathrm{H} \alpha$ absorption. As indicated by both panels, spectra of the Type IIn SN 2005cp do not exhibit these deep, broad features, but they are present in the spectra of SN 1987A-like SNe. We note that the spectrum of SN 2005cp plotted for comparison with the VLT spectrum does exhibit a possible feature near $\mathrm{Na} I \mathrm{D}$, although it shows a different shape and its minimum is not as deep as that of SN 2006V. The spectrum of SN 2005cp taken at an earlier phase than what is plotted for comparison with the grism spectrum exhibits no similar potential feature near Na I D. Strong Ba II (including 5854, 6142, $6497 \AA$ A) absorption features were present in spectra of SN 1987A (see Figure 6) and are found in spectra of underluminous SNe II (Pastorello et al. 2004). SN Refsdal is a comparatively luminous SN 1987A-like SN and does not show strong evidence for Ba II absorption features.

To constrain the source of the ionizing radiation and properties of the emitting gas, we construct a Baldwin, Phillips, $\&$ Terlevich (BPT; Baldwin et al. 1981) diagram, plotted in Figure 15. The intensity ratios [O III] $\lambda 5007 / \mathrm{H} \beta$ and [N II] $\lambda 6584 / \mathrm{H} \alpha$ of the strong emission lines from near the SN site and the nuclear region are consistent with the ratios expected for gas ionized by radiation from massive stars, and the positions on the BPT diagram coincide with the Sloan Digital Sky Survey (SDSS; Thomas et al. 2013) star-forming galaxy population.

We next estimate the reddening along the line of sight to the emitting gas from the measured Balmer decrement. Making the assumption of Case B recombination, we apply the prescription from Domínguez et al. (2013) and adopt the $R_{V}=2.51$ Reddy et al. (2015) extinction curve inferred from MOSFIRE Deep Evolution Field spectroscopy of galaxies at $z \approx 1.4-2.6$. We estimate a color excess of $E(B-V)=0.1 \pm 0.2 \mathrm{mag}$
$\left(A_{V}=0.3 \pm 0.4 \mathrm{mag}\right)$ for the nuclear region and $E(B-V)=0.8 \pm 0.4 \mathrm{mag}\left(A_{V}=2.0 \pm 1.0 \mathrm{mag}\right)$ near the $\mathrm{SN}$ site. In Table 10, we also list the extinction expected for $\mathrm{H} \alpha$.

SN Refsdal occurred at an offset from its host galaxy's nucleus of $\sim 7 \mathrm{kpc}$, and Yuan et al. (2015) used OSIRIS (Larkin et al. 2006) integral-field-unit observations to constrain the oxygen abundance at $\sim 5-7 \mathrm{kpc}$ to be $12+\log (\mathrm{O} / \mathrm{H})_{\mathrm{PP} 04 \mathrm{~N} 2} \leqslant$ 8.11 dex by comparing an upper limit on the [N II] emission to the measured $\mathrm{H} \alpha$ flux using the Pettini \& Pagel (2004) abundance diagnostic. As in Yuan et al. (2015), we use the Pettini \& Pagel (2004) N2 metallicity indicator based on the intensity ratio [N II] $\lambda 6584 / \mathrm{H} \alpha$. We find that the nuclear region has an oxygen abundance of $12+\log (\mathrm{O} / \mathrm{H})=8.6 \pm 0.1$ dex. From an upper limit on the [N II] flux, we compute a $3 \sigma$ upper limit of 8.4 dex near the SN site. The MOSFIRE spectrum yields a $3 \sigma$ detection of $[\mathrm{N} \mathrm{II}]$ and an estimate for the oxygen abundance of $12+\log (\mathrm{O} / \mathrm{H})=8.3 \pm 0.1 \mathrm{dex}$. The presence of strong night-sky lines close to both [N II] emission lines limits the sensitivity of the spectra to the metallicity. Since the ratio of nitrogen to oxygen and the ionization parameter substantially affect the N2 metallicity diagnostic, our estimates may have an accuracy of only $\sim 0.2$ dex. The relatively large uncertainty in our measurement of $\mathrm{H} \beta$ implies that the $R_{23}$ diagnostic (Pagel et al. 1979) is not able to provide a useful constraint on the oxygen abundance.

Karman et al. (2016) have used the Multi Unit Spectroscopic Explorer on the VLT to take optical spectra of the three separate images of the host-galaxy environment of SN Refsdal. Within a 0 " 6 -radius aperture surrounding the explosion site, they report a high [O II]/Mg II ratio of 10-20, consistent with low metallicity and a high ionization parameter. From the $\mathrm{X}$-shooter spectra, we measure $3 \sigma$ limits on the flux of $\mathrm{Mg}$ II $\lambda 2796$ to be $\leqslant 4.7 \times 10^{-18} \mathrm{erg} \mathrm{s}^{-1} \mathrm{~cm}^{-2}$ and of $\mathrm{Mg}$ II $\lambda 2803$ to be $\leqslant 6.3 \times 10^{-18} \mathrm{erg} \mathrm{s}^{-1} \mathrm{~cm}^{-2}$, consistent with the flux levels measured by Karman et al. (2016).

\subsection{Comparison of Host-galaxy Environments}

The low oxygen abundance inferred for the explosion environment of SN Refsdal is consistent with a preference for low metallicity among low-redshift SN 1987A-like SNe. Taddia et al. (2013) reported an average value of $12+\log$ $(\mathrm{O} / \mathrm{H})=8.36 \pm 0.05 \mathrm{dex}(\mathrm{PP} 04 \mathrm{~N} 2)$ for the local environments of SN 1987A-like SNe. They found that these abundances were more metal-poor than those of SN IIP environments (within $\sim 3 \mathrm{kpc}$ ) measured by Anderson et al. (2010). Additional circumstantial evidence for low-metallicity environments include a preference for low-mass dwarf host galaxies, or the periphery of late-type spirals (Pastorello et al. 2012). By contrast, normal SNe II trace the light $g$-band light of their host galaxies (Kelly et al. 2008) and show no preference for metal-poor galaxies or the peripheries of their host galaxies (Kelly \& Kirshner 2012).

\section{SUMMARY}

We have used HST WFC3 grism and VLT X-shooter NIR spectra and images to classify SN Refsdal, the first example of a resolved, strongly lensed SN. Its slowly rising broad-band HST light curve can be matched by those of SN 1987A-like $\mathrm{SNe}$, a peculiar class of SNe II that may account for $\sim 1.5 \%-$ $3 \%$ of nearby SNe (Smartt et al. 2009; Kleiser et al. 2011; 

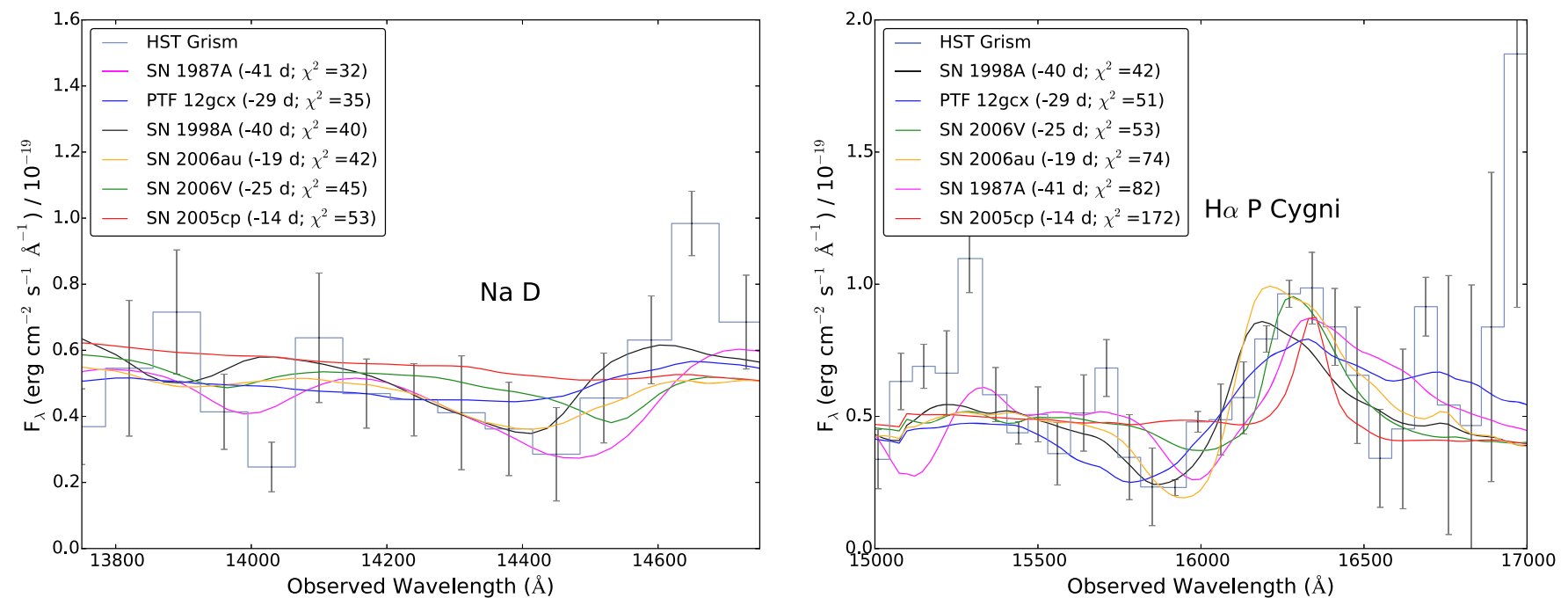

Figure 8. Fits of the Na I D and H $\alpha$ spectral regions of the HST WFC3 G141 spectrum of SN Refsdal ( $-47 \pm 8$ days) using spectra of SN 1987A-like SNe as well as SN 2005cp, a probable SN 1987A-like SN with a dense, H-rich CSM. Spectra of SN 1987A-like SNe without dense CSM exhibit strong, broad Na I D and H $\alpha$ absorption features, and these templates provide much better models of these spectral regions than a spectrum of the Type In SN $2005 \mathrm{cp}$. Here we find the best $\chi^{2}$ agreement with each template spectrum by separately varying the normalization of the template across the wavelength ranges $13750-14750 \AA$ (Na I D) and $15500-16600 \AA(\mathrm{H} \alpha)$. Interpretation of $\chi^{2}$ values on the basis of Kullback-Leibler information entropy (Akaike 1974; Sugiura 1978) indicates that a $\chi^{2}$ difference of 2 is evidence against the more poorly fitting model and 6 constitutes strong positive evidence (e.g., Kass \& Raftery 1995; Mukherjee et al. 1998). The grism data disfavor the lack of a strong, broad Na I D absorption feature and the relatively narrow H $\alpha$ profiles of SN 2005cp, which was likely a SN 1987A-like SN erupting into dense CSM.

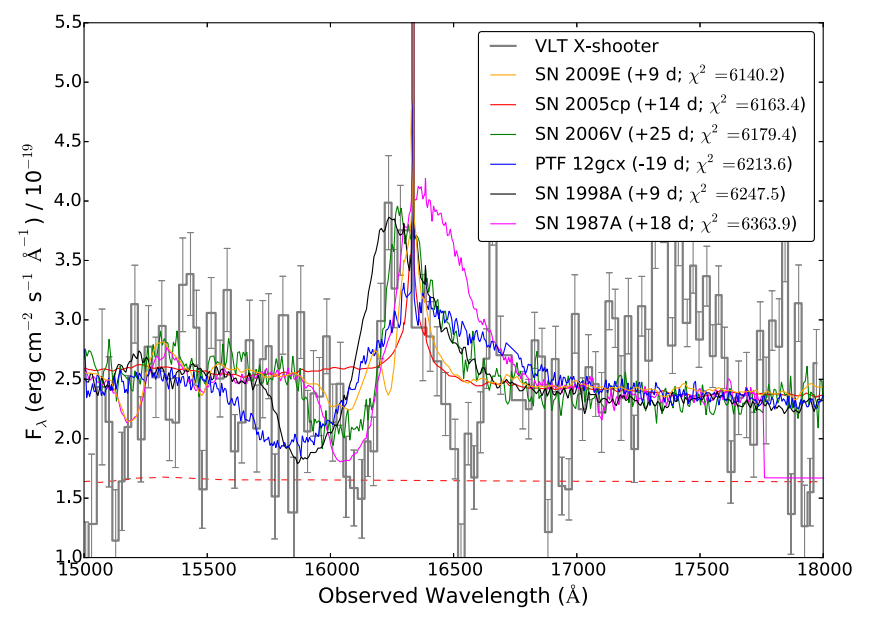

Figure 9. Comparison between the VLT X-shooter spectrum of $\mathrm{H} \alpha$ taken $+16 \pm 8$ days relative to maximum light and the spectra of SN 1987A-like $\mathrm{SNe}$ taken at a roughly similar phase. Here we limit the comparison to a spectral region near the broad $\mathrm{H} \alpha$ line. We compute the $\chi^{2}$ agreement between the $\mathrm{X}$-shooter spectrum and the comparison spectra. Interpretation of $\chi^{2}$ values on the basis of Kullback-Leibler information entropy (Akaike 1974; Sugiura 1978 ) indicates that a $\chi^{2}$ difference of 2 is evidence against the more poorly fitting model and 6 constitutes strong positive evidence (e.g., Kass \& Raftery 1995, Mukherjee et al. 1998). The VLT data disfavor the relatively narrow $\mathrm{H} \alpha$ profiles of SN 2005cp, a SN 1987A-like SN that was classified as a SN IIn. For this comparison, we mask out data within $10 \AA$ of strong nebular emission lines.

Pastorello et al. 2012). The only other SNe having similar light curves and colors are SNe IIn, which erupt into dense CSM and whose light curves are heterogeneous. Detection of strong and wide Na I D absorption and $\mathrm{H} \alpha$ P-Cygni absorption in the SN Refsdal spectra, however, identifies it as a SN 1987A-like SN, and provides strong evidence against the possibility that SN Refsdal is a SN IIn with continuum emission arising primarily from circumstellar interaction near peak luminosity.

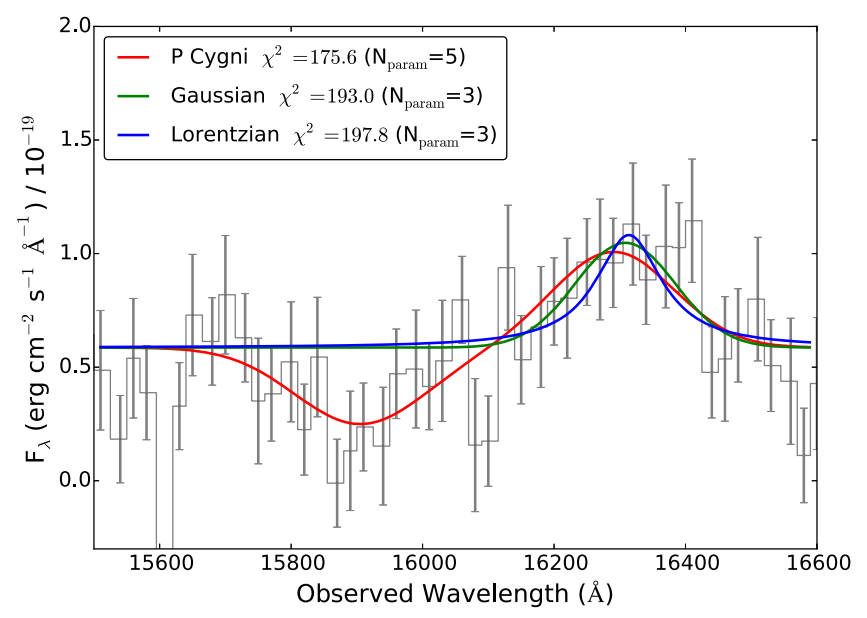

Figure 10. Model fits to the HST WFC3 G141 grism spectrum of $\mathrm{H} \alpha$ taken $-47 \pm 8$ days relative to maximum light. Table 5 lists the changes in the AIC between the model fits. While the $\chi^{2}$ values listed are from fitting the unbinned spectra, we plot the spectra after binning. The best-fitting Gaussian model has $\sigma=90 \AA\left(1650 \mathrm{~km} \mathrm{~s}^{-1}\right)$. The data plotted above are in bins of $20 \AA$.

The specific properties of the $\mathrm{H} \alpha$ emission and absorption features are also consistent with measurements of low-redshift SN 1987A-like SNe. The $\mathrm{H} \alpha$ expansion velocity we measure from the grism spectrum agrees with the velocities of SN 1987A-like SNe at comparable pre-maximum phases. Similarly, the strength and evolution of the $\mathrm{H} \alpha$ luminosity are consistent with those of SN 1987A-like events, and may not have been as strong or evolved the same way as was observed for the Type IIn SN 2005cp. Finally, the $\mathrm{H} \alpha$ emission-line profile is Gaussian instead of Lorentzian.

SN 1987A was the nearest detected SN of the modern era and is the best-studied event. Its progenitor star ( $\left.\mathrm{Sk}-69^{\circ} 202\right)$ in the LMC was a compact blue supergiant (B3 I; Gilmozzi et al. 1987; Sonneborn et al. 1987) inferred to have a mass 


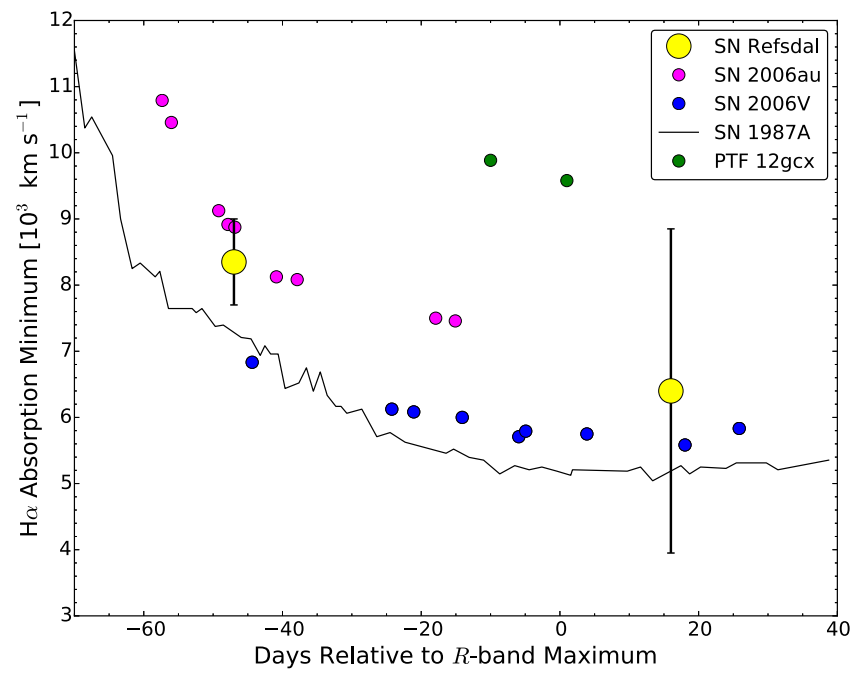

Figure 11. Comparison between constraints on $\mathrm{H} \alpha$ P-Cygni absorption expansion velocity and $\mathrm{H} \alpha$ expansion velocities of SN 1987A-like SNe. The plotted measurements of SN 2006V, SN 2006au, and SN 1987A are from Taddia et al. (2012). The early-time $\mathrm{H} \alpha$ expansion velocity of SN Refsdal is from the WFC3 grism spectra, and the later constraint is from the X-shooter spectra.

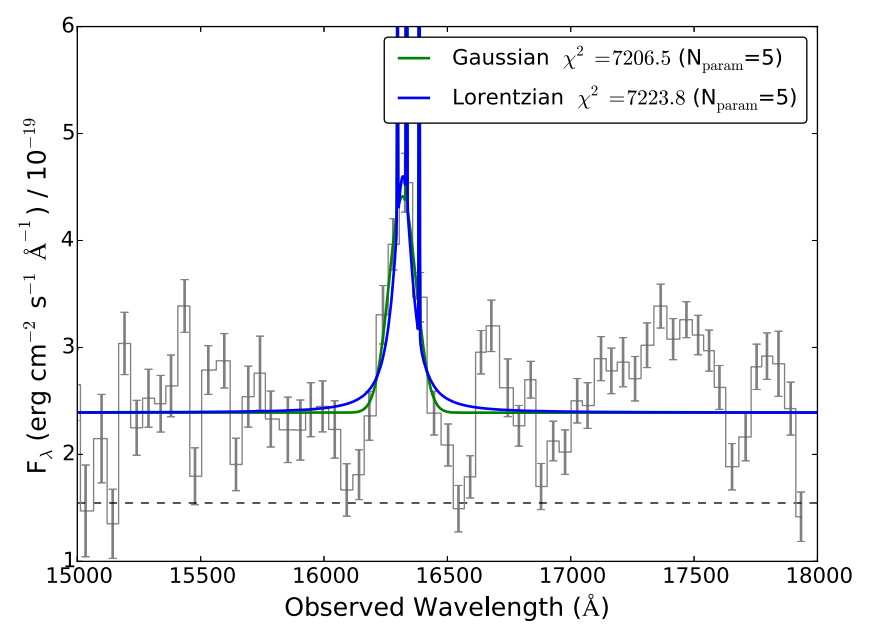

Figure 12. Model fits to the VLT X-shooter spectrum of $\mathrm{H} \alpha$ taken $+16 \pm 8$ days relative to maximum light at the position of SN Refsdal. Table 5 lists the changes in the AIC between the model fits. While the $\chi^{2}$ values listed are from fitting the unbinned spectra, we plot the spectra after binning. The best-fitting Gaussian model has $\sigma=55 \AA\left(1000 \mathrm{~km} \mathrm{~s}^{-1}\right)$.

close to $20 M_{\odot}$ (see Arnett et al. 1989 for a review). The size of the progenitor $\left(\lesssim 100 R_{\odot}\right)$ was significantly smaller than those of average red supergiants $\left(500-1000 R_{\odot}\right)$, and accounts for the slowly rising light curve. Pastorello et al. (2012) suggest that all known SN 1987A-like SNe are consistent with explosions of blue supergiant progenitors with radii between 35 and $90 R_{\odot}$ and masses at explosion of $\sim 20 M_{\odot}$, which would be higher, on average, than those identified for SN IIP progenitors (Smartt et al. 2009).

For assumed values of the magnification, SN Refsdal had comparatively luminous $M_{B}$ and $M_{V}$ absolute magnitudes relative to well-studied low-redshift SN 1987A-like SNe (Pastorello et al. 2005; Kleiser et al. 2011; Pastorello et al. 2012; Taddia et al. 2012). While the $K$-corrections and photometric errors introduce some uncertainty, we find that

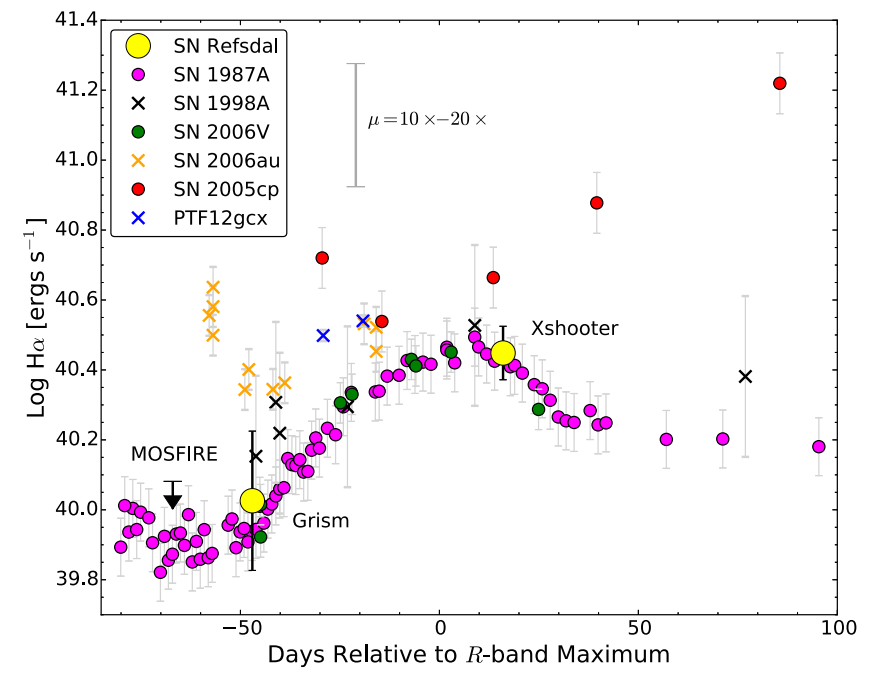

Figure 13. Comparison between the $\mathrm{H} \alpha$ luminosity evolution of SN Refsdal (after correcting for magnification) and that of SNe with SN 1987A-like light curves. The SNe plotted for comparison were spectroscopically classified as Type IIP, with the exception of SN 2005cp. The relatively narrow Balmer emission lines of SN 2005cp led it to be classified as a SN IIn, although its light curve is similar to those of SN 1987A-like SNe. The upper limit on the $\mathrm{H} \alpha$ luminosity obtained $-62 \pm 8$ days before $R$-band maximum is from the MOSFIRE integration, the measurement at $-47 \pm 8$ days is from the WFC3 G141 grism data, and the measurement at $+16 \pm 8$ days is from the $\mathrm{X}$-shooter spectrum. Plotted error bars correspond to uncertainties in the distance to the explosion, or in the case of SN Refsdal, the magnification from the cluster. We have assumed $\mu=15$, and the gray line shows the systematic shift in the inferred $\mathrm{H} \alpha$ line luminosity as the magnification is shifted between $\mu=10$ and $\mu=20$.

Table 7

Best-fitting Template Spectra in the Superfit Library

\begin{tabular}{lcrrc}
\hline \hline Supernova & Type & Phase & $A_{V}$ (mag) & $S$ \\
\hline SN 1998A & II & -40 & 1.1 & 44.62 \\
SN 2005cs & II & +2 & 1.9 & 48.98 \\
SN 2005cs & II & +14 & 0.9 & 49.05 \\
SN 2004et & II & +47 & -0.9 & 49.20 \\
SN 1999em & II & +20 & -0.2 & 49.45 \\
SN 1986I & II & +83 & 1.1 & 49.70 \\
SN 2004et & II & +45 & -0.7 & 49.79 \\
SN 2005cs & II & +1 & 1.9 & 49.90 \\
SN 2005cs & II & +6 & 0.9 & 49.98 \\
SN 2005cs & II & +11 & 0.5 & 50.45 \\
SN 1999em & II & +9 & -0.2 & 50.48 \\
SN 1999em & II & +15 & -0.4 & 50.50 \\
SN 1999em & II & +4 & 1.7 & 50.79 \\
theory99em & II & +25 & -0.7 & 50.85 \\
SN 1993W & II & +21 & -0.1 & 50.85 \\
\hline
\end{tabular}

Note. The fifteen best-matching Superfit (Howell et al. 2005) templates are SNe II showing P-Cygni profiles, which are characterized by the presence of $\mathrm{H}$ features. $S=\Sigma\left(F_{i}-T_{i} \times 10^{-A_{\lambda} / 2.5}\right) / \sigma_{i}^{2}$, where $F_{i}$ is the $F_{\lambda}$ flux of the SN measured in each resolution element, $\sigma_{i}$ is the uncertainty of the measured flux, $T_{i}$ is the flux of template spectrum, and $A_{\lambda}$ is the wavelength-dependent extinction for an $R_{V}=3.1$ law. The normalization of the template spectrum and the extinction $A_{V}$ are the fitting parameters. We fix the redshift to be that of the host galaxy $(z=1.49)$.

the $V-R$ color of SN Refsdal may be similar to those of blue low-redshift SN 1987A-like SNe, but that it may have an exceptionally blue $B-V$ color, especially near maximum light. Scaling from a model of SN 1987A and using the fact 
Table 8

Host-galaxy Emission Lines

\begin{tabular}{lc}
\hline \hline Line & Flux \\
& $\left(10^{-17} \mathrm{erg} \mathrm{s}^{-1} \mathrm{~cm}^{-2}\right)$ \\
\hline$[\mathrm{N}$ II] $\lambda 6548$ & $0.197 \pm 0.0598$ \\
$\mathrm{H} \alpha$ & $5.87 \pm 0.132$ \\
{$[\mathrm{~N} \mathrm{II}] \lambda 6584$} & $0.592 \pm 0.179$ \\
{$[\mathrm{~S} \mathrm{II}] \lambda 6716$} & $1.34 \pm 0.141$ \\
{$[\mathrm{~S} \mathrm{II}] \lambda 6731$} & $0.854 \pm 0.147$ \\
\hline
\end{tabular}

Note. Keck-I MOSFIRE measurements of emission lines and uncertainties. Fluxes are not corrected for extinction or magnification.

Table 9

VLT X-shooter Host-galaxy Emission-line Measurements

\begin{tabular}{|c|c|c|c|}
\hline Source & Line & $\begin{array}{l}\text { Line Flux } \\
\left(10^{-17} \mathrm{erg} \mathrm{s}^{-1} \mathrm{~cm}^{-2}\right)\end{array}$ & $\begin{array}{l}\text { FWHM } \\
\left(\mathrm{km} \mathrm{s}^{-1}\right)\end{array}$ \\
\hline $\mathrm{SN}$ & [O II] $\lambda 3726$ & $0.47 \pm 0.08$ & $43 \pm 18$ \\
\hline $\mathrm{SN}$ & [O II] $\lambda 3729$ & $0.63 \pm 0.01$ & $\ldots$ \\
\hline $\mathrm{SN}$ & $\mathrm{H} \beta$ & $0.2 \pm 0.1$ & $64 \pm 16$ \\
\hline SN & [O III] $\lambda 4959$ & $0.25 \pm 0.05$ & $60 \pm 14$ \\
\hline $\mathrm{SN}$ & [O III] $\lambda 5007$ & $0.7 \pm 0.1$ & $\ldots$ \\
\hline $\mathrm{SN}$ & $\mathrm{H} \alpha$ & $1.5 \pm 0.3$ & $66 \pm 11$ \\
\hline $\mathrm{SN}$ & [N II] $\lambda 6584$ & $\leqslant 0.24$ & $62 \pm 13$ \\
\hline Host nucleus & [O II] $\lambda 3726$ & $3.1 \pm 0.2$ & $185 \pm 18$ \\
\hline Host nucleus & {$[\mathrm{O}$ II] $\lambda 3729$} & $3.6 \pm 0.2$ & $\ldots$ \\
\hline Host nucleus & $\mathrm{H} \beta$ & $4.4 \pm 0.6$ & $168 \pm 7$ \\
\hline Host nucleus & [O III] $\lambda 4959$ & $0.9 \pm 0.2$ & $177 \pm 20$ \\
\hline Host nucleus & [O III] $\lambda 5007$ & $1.0 \pm 0.2$ & $\ldots$ \\
\hline Host nucleus & $\mathrm{H} \alpha$ & $14.0 \pm 0.2$ & $203 \pm 30$ \\
\hline Host nucleus & {$[\mathrm{N} \mathrm{II}] \lambda 6584$} & $4 \pm 1$ & $187 \pm 18$ \\
\hline
\end{tabular}

Note. Measurements of emission lines and uncertainties. Fluxes are not corrected for extinction or magnification. FWHM line widths are corrected for the instrumental resolution. For the instrumental resolution, the nominal value for the $0 . ! 6$ slit is used.

that SN 1987A-like SNe exhibit a relatively small range of rise times, we estimate an ejecta mass of $20 \pm 5 M_{\odot}$ for the explosion.

We note that circumstellar interaction can range in intensity from very weak to very strong depending on the distribution, mass, and composition of any material surrounding the star. In the case of PTF 11iqb, for example, the CSM was overtaken quickly by the expanding ejecta and early-time narrow lines disappeared (Smith et al. 2015). Despite the lack of narrow lines near maximum light in spectra of PTF 11iqb, the CSM likely caused the SN to have a bluer color and higher luminosity. Indeed, SN 1987A is surrounded by a ring thought to have been ejected $\sim 10^{4}$ years before the explosion (Meaburn et al. 1995; Crotts \& Heathcote 2000). Smith et al. (2014) have found that the light curves and spectra of the Type IIn SN 2009ip and SN 2010mc could be explained by the faint SN 1987A-like explosions of blue supergiant progenitors into dense CSM. However, deep and broad observed $\mathrm{NaID}$ and $\mathrm{H} \alpha$ absorption features, the measured $\mathrm{H} \alpha$ evolution and strength, and the SN luminosity indicate that circumstellar interaction is not responsible for a substantial fraction of the SN Refsdal's luminosity during its photospheric phase.
Table 10

Host-galaxy Measurements

\begin{tabular}{lccc}
\hline \hline Measurement & Nuclear Region & SN Site & Instrument \\
\hline $\begin{array}{c}12+\log (\mathrm{O} / \mathrm{H}) \\
(\mathrm{PP} 04 \mathrm{~N} 2)\end{array}$ & $\ldots$ & $8.3 \pm 0.1 \mathrm{dex}$ & MOSFIRE \\
$12+\log (\mathrm{O} / \mathrm{H})$ & $8.6 \pm 0.1 \mathrm{dex}$ & $8.4 \mathrm{dex}(3 \sigma$ upper & X-shooter \\
$(\mathrm{PP} 04 \mathrm{~N} 2)$ & limit $)$ & \\
$E(B-V)$ & $0.1 \pm 0.2 \mathrm{mag}$ & $0.8 \pm 0.4 \mathrm{mag}$ & X-shooter \\
$A_{\mathrm{H} \alpha}$ & $0.2 \pm 0.3 \mathrm{mag}$ & $1.5 \pm 0.9 \mathrm{mag}$ & X-shooter \\
$A_{V}$ & $0.3 \pm 0.4 \mathrm{mag}$ & $2.0 \pm 1.0 \mathrm{mag}$ & X-shooter \\
\hline
\end{tabular}

Note. Properties of the ionized gas near the SN explosion site and in the hostgalaxy nuclear region, as well as reddening along the line of sight from analysis of strong nebular emission lines.

Ongoing late-time HST imaging of the four images of SN Refsdal (PI P. Kelly; GO-14199) will continue to improve our understanding of the SN. As predicted (Diego et al. 2015; Grillo et al. 2016; Jauzac et al. 2015; Kawamata et al. 2015; Kelly et al. 2015b; Oguri 2015; Sharon \& Johnson 2015; Treu et al. 2016), SN Refsdal has reappeared in a different image of its spiral host galaxy (Kelly et al. 2015a). While other faded SNe depart forever, we will have a second opportunity to study SN Refsdal and to learn about its early evolution.

We would like to thank Space Telescope Science Institute (STScI) director Matt Mountain for making it possible to obtain the HST WFC3 grism spectra of SN Refsdal. We express our appreciation for the efforts of HST Program Coordinator Beth Periello and Contact Scientist Norbert Pirzkal, as well as Claus Leitherer, Andrew Fox, Ken Sembach, and Miranda Link for scheduling the grism observations. Based in part on observations made with ESO telescopes at the La Silla Paranal Observatory under program ID 295.D-5014. We thank Ori Fox for helpful discussions about the Type IIn SN 2005cp, and Nathan Smith for useful comments about CSM interaction and SN 1987A-like SNe.

Support for the preparation of the paper is from HST grants GO-14041 and GO-14199. The GLASS program was supported by GO-13459, and the FrontierSN photometric followup program has funding through GO-13386. A.Z. is supported by a Hubble Fellowship (HF2-51334.001-A) awarded by STScI, which is operated by the Association of Universities for Research in Astronomy, Inc., for NASA, under contract NAS 5-26555. R.J.F. gratefully acknowledges support from National Science Foundation (NSF) grant AST-1518052 and the Alfred P. Sloan Foundation. Supernova research at Rutgers University (S.W.J.) is supported by NSF CAREER award AST-0847157, and NASA/Keck JPL RSA 1508337 and 1520634. C.M. is supported through NSF grant AST1313484. The DNRF provided funding for the Dark Cosmology Centre. JMS is supported by an NSF Astronomy and Astrophysics Postdoctoral Fellowship under award AST1302771. A.V.F.'s group at UC Berkeley has received generous financial assistance from the Christopher R. Redlich Fund, the TABASGO Foundation, Gary \& Cynthia Bengier, and NSF grant AST-1211916. The work of A.V.F. was completed at the Aspen Center for Physics, which is supported by NSF grant PHY-1066293; he thanks the Center for its hospitality during the black holes workshop in 2016 June and July. 


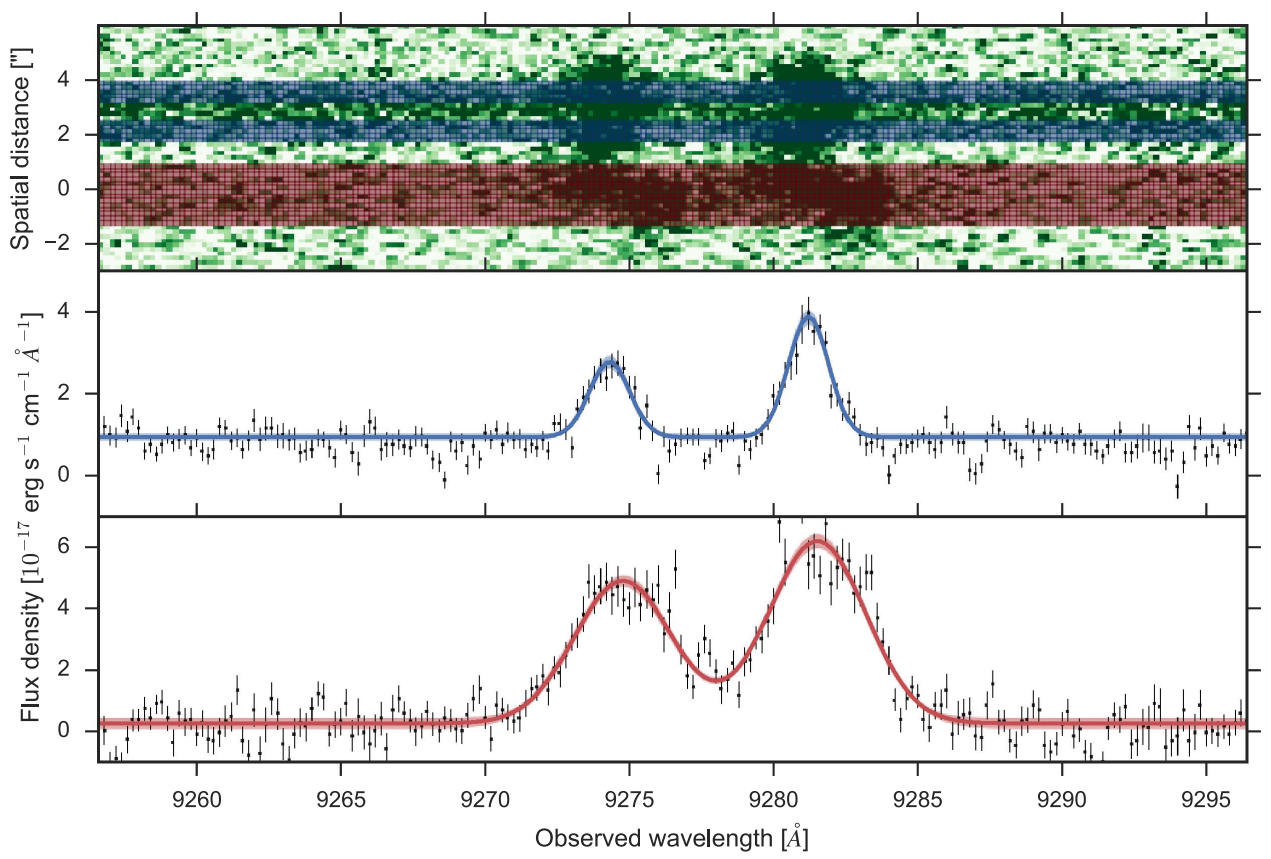

Figure 14. Example of nebular emission line detection in VLT/X-shooter spectra. The top panel is the two-dimensional spectrum from OB2 (see Figure 1) in the region surrounding $[\mathrm{O} \mathrm{II}] \lambda \lambda 3726,3729$. The ordinate corresponds to the spatial direction and the abscissa is the spectral direction. The blue-colored regions are the extraction apertures used for the position of the SN images S1 and S2 (above and below, respectively). The trace visible between the two SN positions is light from the early-type galaxy lens. The red-colored region is the aperture used to extract the spectrum of the host nucleus. The [O II] $\lambda \lambda 3726,3729$ doublet is cleanly resolved in the X-shooter spectrum, and significant structure is visible in both the spatial and dispersion directions. The middle panel shows the fit to the [O II] $\lambda \lambda 3726,3729$ emission lines at the SN positions, while the bottom panel plots the same for the host nucleus.

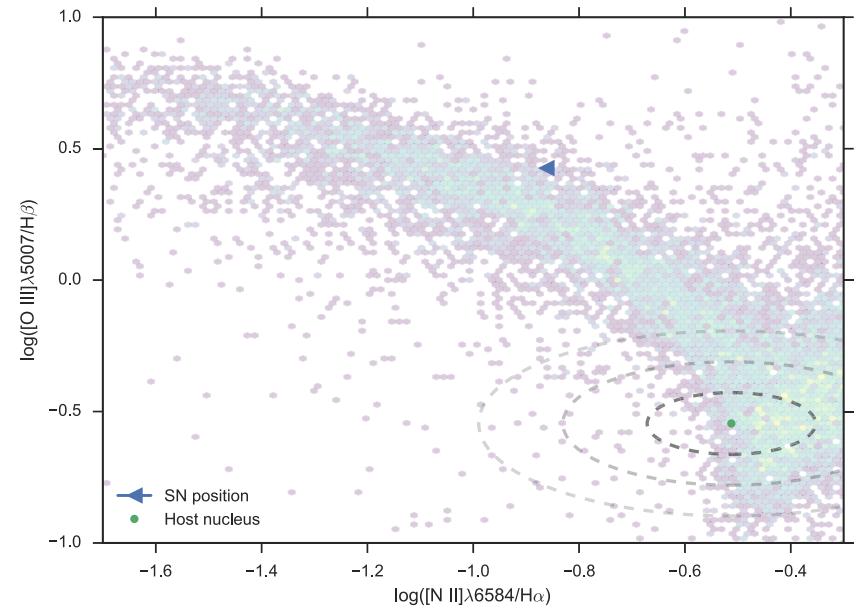

Figure 15. BPT diagram (Baldwin et al. 1981) for the emission lines measured from two host-galaxy extractions. The green dot shows the ratios for the host nuclear region, where the ellipses correspond to $1 \sigma, 2 \sigma$, and $3 \sigma$ confidence contours. The blue triangle marks the ratios of emission lines originating from near the SN position, where only an upper limit for [N II] flux could be estimated. This turns into an upper limit on the $\log ([\mathrm{N} \mathrm{II}] / \mathrm{H} \alpha)$ ratio. Underplotted is the same ratio for all the $z \leqslant 0.1$ objects in the SDSS DR12 (Thomas et al. 2013).

This research uses services or data provided by the NOAO Science Archive. NOAO is operated by the Association of Universities for Research in Astronomy (AURA), Inc., under a cooperative agreement with the NSF. Some of the data presented herein were obtained at the W. M. Keck Observatory, which is operated as a scientific partnership among the California Institute of Technology, the University of California, and NASA; the observatory was made possible by the generous financial support of the W. M. Keck Foundation. We recognize the Hawaiian community for the opportunity to conduct these observations from the summit of Mauna Kea.

\section{APPENDIX: METHODS}

\section{A.1. Phases of the MOSFIRE, WFC3, and $X$-shooter Spectra Relative to Time of Maximum Light}

The mean phase relative to maximum light (in the $\mathrm{SN}$ rest frame) of the Keck-I MOSFIRE data is $-62 \pm 8$ days, of the HST WFC3 G141 grism spectra is $-47 \pm 8$ days, and of the VLT $\mathrm{X}$-shooter spectra is $+16 \pm 8$ days. At maximum light, $F 160 W \approx 24.45$ mag AB for image S2.

\section{A.2. HST WFC3-IR G141 Grism Data}

\section{A.2.1. Processing}

We processed the WFC3/IR FLT images obtained from the $H S T$ archive using the software pipeline developed for the 3D$H S T$ project $^{25}$ (Brammer et al. 2012b). This pipeline employs the DrizzlePac package (Gonzaga 2012) ${ }^{26}$ to align the WFC3 images, and a custom software package to extract and model the grism spectra (Brammer et al. 2012a; Momcheva et al. 2015). ${ }^{27,28}$

\footnotetext{
25 https://github.com/gbrammer/threedhst

26 http://adsabs.harvard.edu/abs/2012drzp.book.....G

27 http://adsabs.harvard.edu/abs/2012ApJ...758L..17B

${ }^{28}$ http://adsabs.harvard.edu/abs/2015arXiv151002106M
} 

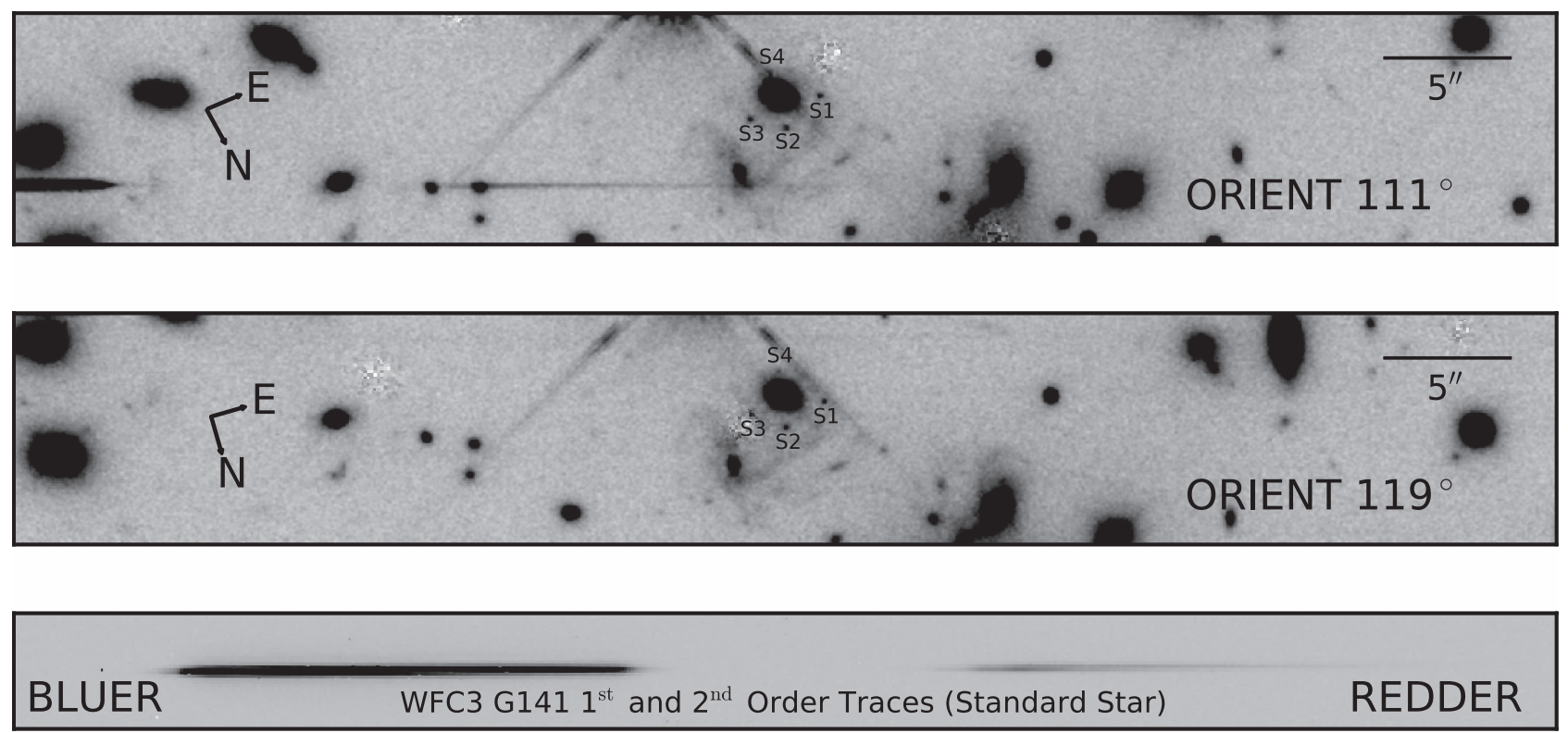

Figure 16. Coaddition of WFC $3 / \mathrm{IR} F 125 \mathrm{~W}$ and $F 160 \mathrm{~W}$ exposures taken before G141 grism integrations at the same orientation. The dispersion axis is horizontal. Unlike with a prism, blue light makes a smaller relative angle with the grism. The first-order trace spans $11000-17000 \AA$ ( $~ 4400-6800 \AA$ in the SN rest frame). Bottom panel shows the first- and second-order traces in an observation of the standard star GD-153 (GO-13092; PI J. Lee).

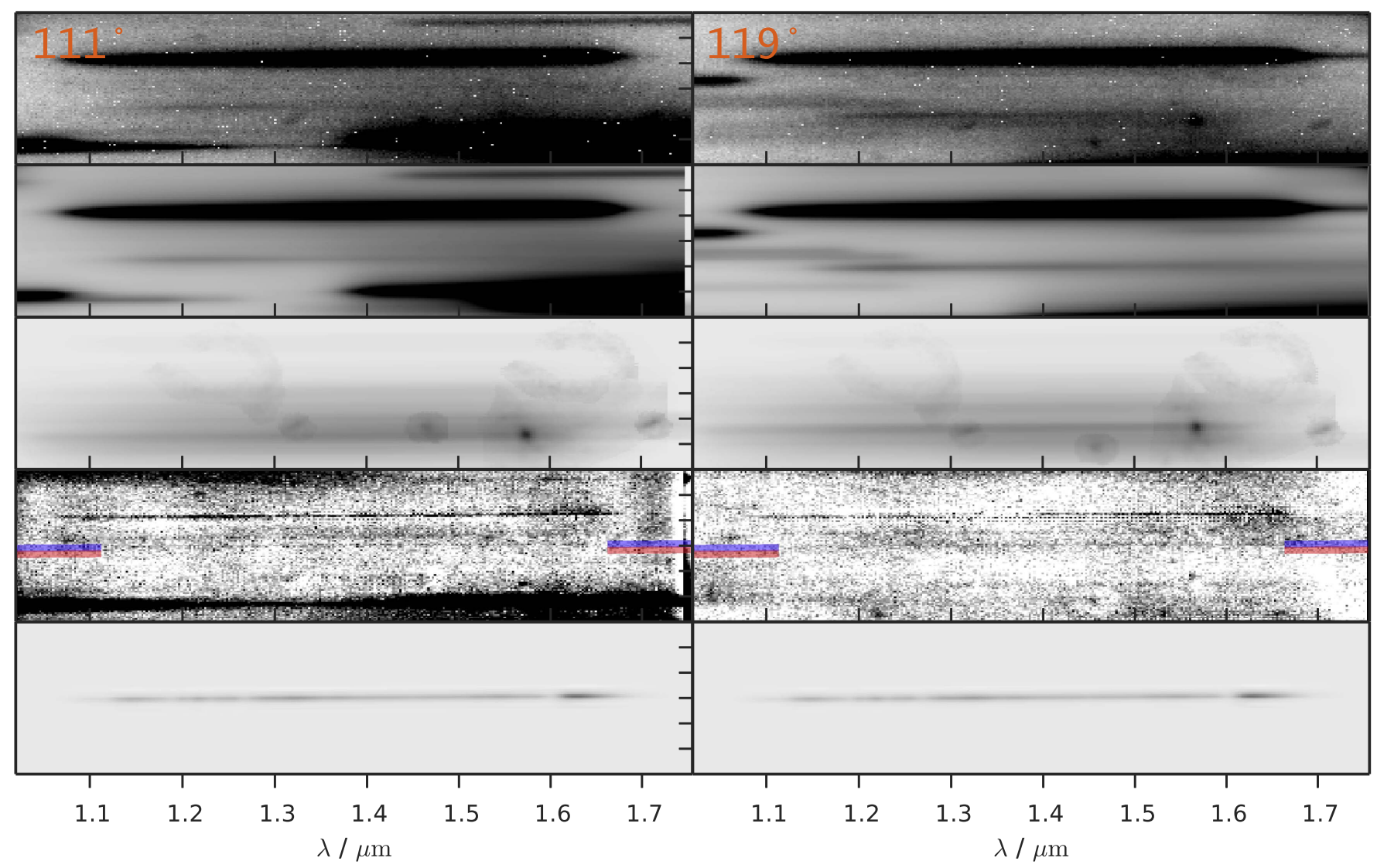

Figure 17. WFC3 G141 two-dimensional grism spectra taken of SN Refsdal in the $111^{\circ}$ and $119^{\circ}$ orientations. For each orientation (beginning from the top), the first panel shows the full observed spectrum, the second panel gives a model of nearby contaminants, the third panel provides the model of the H $\alpha$ and [O III] emission lines, and the fourth panel is the cleaned residual spectrum, with the stretch now 10 times that of the preceding panels. The final panel is the model two-dimensional spectrum of the SN IIP template. Blue bands show the extraction region of the S2 trace itself (it continues across the whole spectrum but is only shown at the edges, so one can actually see the spectrum under it) and the red band is the adjacent aperture used for the local background. The tick marks on the ordinate axis are spaced by $1 . "$ 

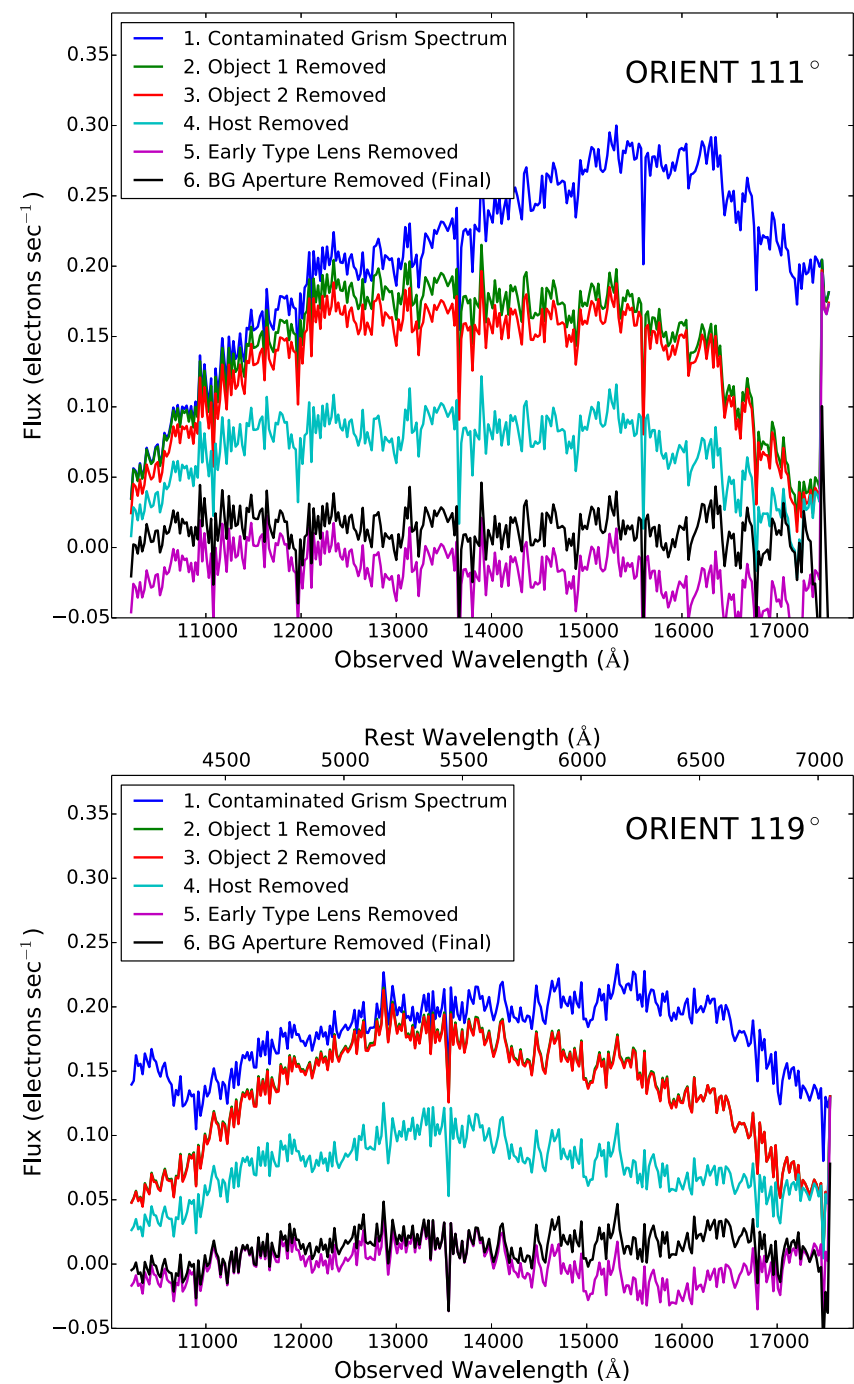

Figure 18. Successive subtraction of contamination along the WFC3 grism trace of SN Refsdal taken in the $111^{\circ}$ (top panel) and $119^{\circ}$ (bottom) telescope orientations. The light from SN Refsdal constitutes only $10 \%-15 \%$ of the light in the extraction aperture. The steps follow in parallel the order of panels from top to bottom in Figure 17. The background subtraction ("BG Aperture") is the flux measured in an aperture running parallel to the extraction aperture. Background removal corrects a modest overcorrection for contamination at redder wavelengths. Despite extremely strong contamination, synthetic magnitudes of the subtracted spectrum yield a $F 125 W-F 160 W$ color that has reasonable agreement with magnitudes measured from direct imaging (see Appendix A.2.4).

\section{A.2.2. Modeling Contaminant Spectroscopic Traces}

In a grism spectral element, light passes first through a prism and then a diffraction grating. The light from all objects in the field is dispersed in a common direction, making efficient spectroscopy of a large number of objects possible. The Einstein cross, however, presents a challenge for grism spectroscopy, because the SN images are embedded in light from both the spiral host galaxy and the early-type galaxy lens. In Figure 16, we show a coaddition of all $F 125 W$ and $F 160 W$ direct exposures of the $\mathrm{SN}$ images $\mathrm{S} 1-\mathrm{S} 4$, adjacent sources, and the dimensions of the first-order and second-order grism traces. The traces of nearby, bright cluster members create additional strong contamination, and a nearby bright star with $r=15.1 \mathrm{mag} \mathrm{AB}$ produces strong diffraction spikes.
The roll angle of the spacecraft determines the dispersion axis of the WFC3 G141 grism. Acquiring spectra at more than one roll angle makes possible a more robust extraction, because the trace at each roll angle is contaminated by different sources. To identify the two combinations of roll angles and SN images that would yield the least contamination, we simulated the expected grism spectra at all roll angles available at the time of the observation, and predicted that image $\mathrm{S} 2$ observed at angles $111^{\circ}$ and $119^{\circ}$ would be least contaminated.

Even after configuring the observations to obtain the cleanest possible SN spectra, light from other sources accounts for $85 \%-90 \%$ of the light along the traces of image S2 of SN Refsdal. To recover the SN spectrum, we constructed and subtracted models of overlapping traces. We used the wideband $F 125 W-F 160 W$ color and the $F 160 W$ flux to model the continuum of the spiral host galaxy, the $z=0.54$ elliptical cluster-member lens, and the two adjacent red-sequence cluster members. We used the $F 160 \mathrm{~W}$ flux to model the $\mathrm{H} \alpha$ and [O III] narrow-line emission from the $z=1.49$ spiral host galaxy. Figure 17 shows the two-dimensional models for the contaminating sources. Finally, we subtracted the residual background measured within a parallel adjacent aperture. Figure 18 shows the successive removal of the modeled sources and the parallel background measurement for the grism spectrum taken in the $111^{\circ}$ telescope orientation. In Figure 19, we plot the extracted spectra of image S2 in orients $111^{\circ}$ and $119^{\circ}$, and, for comparison, that of SN 1987A at the same epoch.

\section{A.2.3. Rejecting Outlying Flux Measurements}

We compute a smoothed spectrum at the wavelength of each measurement with a weight proportional to a Gaussian density with $\sigma=3000 \mathrm{~km} \mathrm{~s}^{-1}$ in the rest frame and inversely proportional to the square of the uncertainty of the flux measurement (Tonry \& Davis 1979). The narrow lines have already been removed during an earlier processing step.

\section{A.2.4. Comparison Between Direct Imaging Magnitudes and Grism Spectra}

Given the fact that the SN flux was only a small fraction of the contaminating flux along the trace, a useful consistency check is to assess how well synthetic magnitudes calculated from the grism SN spectrum agree with direct photometry of the $\mathrm{SN}$. We compute synthetic $\mathrm{AB}$ magnitudes using the $F 125 W$ and $F 160 W$ filter functions, and calculate a synthetic $F 125 W-F 160 W$ color.

The grism spectra were taken from 2014 December 23 through 2015 January 5, but all of the 202.9 s post-imaging exposures were taken with the $F 160 \mathrm{~W}$ wide-band filter from December 23-28, while all post-imaging exposures from 2014 December 30 through 2015 January 4 were taken with the $F 125 \mathrm{~W}$ wide-band filter. To estimate the average color of SN Refsdal during the period of grism observations, we have coadded post-imaging exposures acquired on 2014 December 28-31, when coverage spans approximately the same epochs. These dates bracket the midpoint of the grism observations on 2014 December 29.

We measure direct magnitudes of $F 125 W=25.01 \pm$ $0.05 \mathrm{mag} \mathrm{AB}$ and $F 160 W=25.06 \pm 0.05 \mathrm{mag}$, or a color of $F 125 W-F 160 W=-0.05 \pm 0.07$ mag AB. Then we calculate a synthetic color of $F 125 W-F 160 W=0.14$ mag AB. 

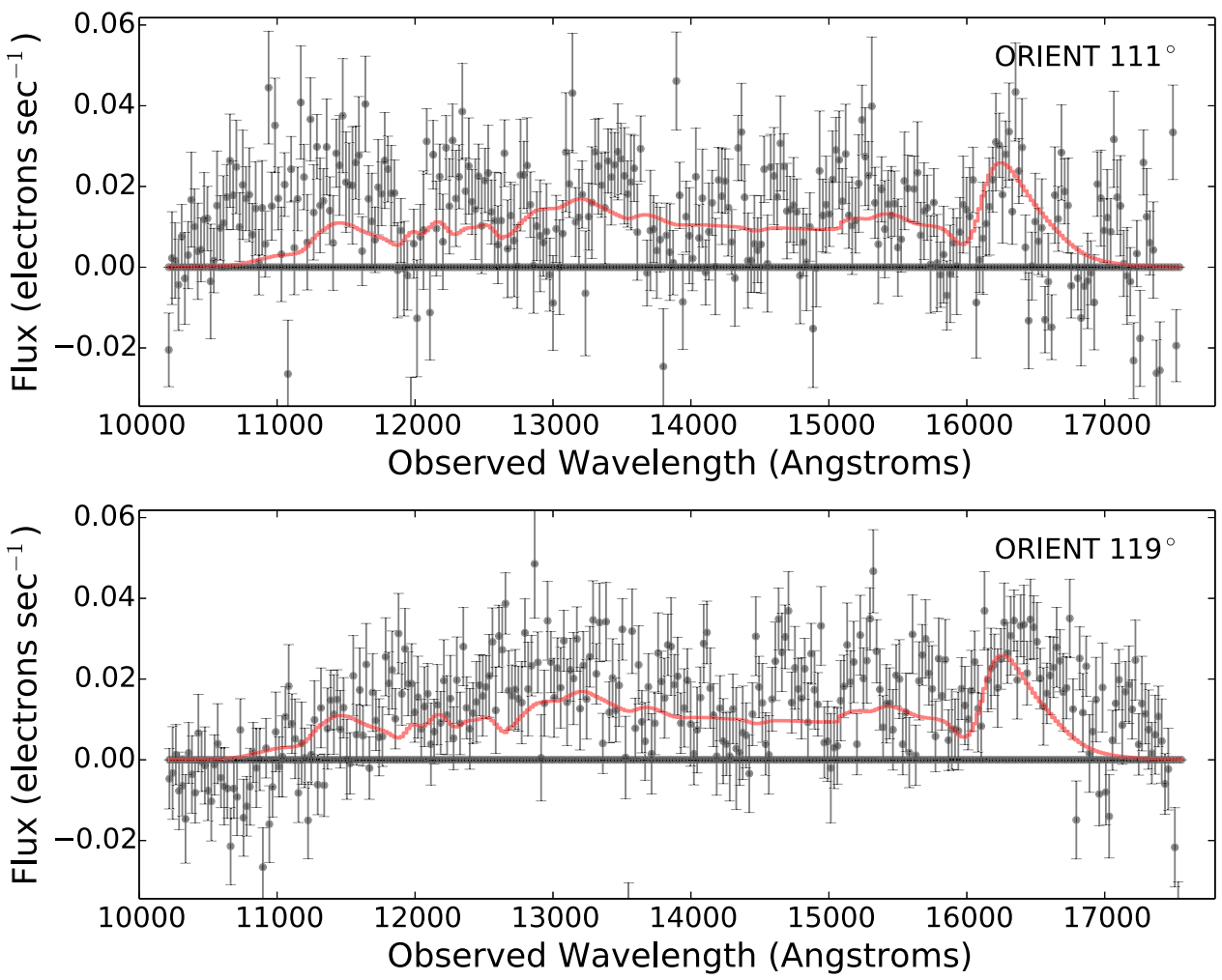

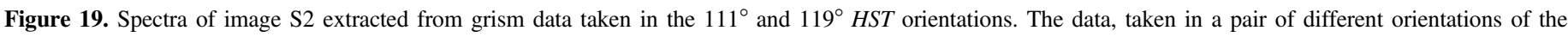

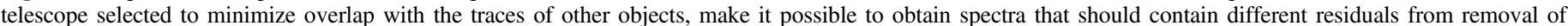
overlapping traces. The superimposed spectrum of SN 1987A is normalized to the average $F 160 W$ magnitude of S2 during the period of observations.

\section{A.3. VLT X-shooter Spectroscopy}

The VLT data were reduced using the ESO/X-shooter pipeline v2.5.2 (Modigliani et al. 2010), where the Reflex interface (Freudling et al. 2013) is used to manage the pipeline. The two-dimensional spectra have been rectified on a grid with $0.2 \AA$ pixel $^{-1}$ in the ultraviolet blue and VIS and $0.6 \AA$ pixel $^{-1}$ in the NIR arm, slightly oversampling the spectra given the nominal X-shooter resolving power. The spectra are flux calibrated using an observation of a spectrophotometric standard (Hamuy et al. 1994; Vernet et al. 2010) from which we measure a response function.

The cluster field in which data were taken contains light from the $z=1.49$ spiral host galaxy and cluster galaxies in the offregion locations observed during the nodding sequence. The continua of these sources are relatively featureless near the $\mathrm{H} \alpha$ emission from the SN, so we do not expect subtraction of the emission from these contaminants to affect the VLT SN spectrum. While there is no evidence of subtraction of significant narrow-line nebular emission for OB1 and OB2, line emission in the "off region" used for OB3 contains strong emission, and we do not use the spectra acquired during this OB when estimating emission-line strengths. A reduction in stare mode has also been carried out, where the sky is estimated using a sigma-clipped mean in the regions free of host-galaxy light. The noise images for both reduction modes are constructed directly from the data at each wavelength bin where the standard deviation is calculated in the regions excluding the host.

The positions of the SN images on the slit were determined by measuring the distance from the host nucleus in the HST image. $\mathrm{H} \alpha$ emission from the host nucleus is clearly visible in the two-dimensional spectrum.

\section{A.3.1. Extraction and Combination}

We have extracted two separate sets of spectra to study the $\mathrm{SN}$ and to examine the strong nebular emission. To study the SN light, we use a 6 pixel (1"!08) wide aperture centered at the positions of the SN. We use a model of sky lines from $\mathrm{ESO}^{29}$ to construct a mask of strong night-sky emission. In addition to line emission, the sky also produces continuum emission, and we model and subtract the continuum emission by computing the median value in bins of $200 \AA$ in wavelength, after removing pixel elements with radiance $>20,000$ photons $\mathrm{s}^{-1} \mathrm{~m}^{-2} \mu \mathrm{m}^{-1} \operatorname{arcsec}^{-2}$. After subtracting the continuum emission from the sky, we interpolate the background-subtracted sky spectrum to the X-shooter wavelength grid and mask pixels with sky values greater than 10,000 photons $\mathrm{s}^{-1} \mathrm{~m}^{-2} \mu \mathrm{m}^{-1} \operatorname{arcsec}^{-2}$ when binning. We also mask any pixels within $6.4 \AA(5 \sigma)$ of a strong nebular emission line, including $\mathrm{H} \alpha$ and [N II]. To study the host-galaxy narrowline emission, we instead extract using an aperture with a width that is $250 \%$ of the average FWHM seeing listed in Table 2.

For both sets of extractions, the flux in each aperture is summed, and the noise spectrum within the same aperture is added in quadrature. We next apply aperture corrections appropriate for the SN point source. To estimate the expected slit loss for the $\mathrm{SN}$ and contamination from the early-type lens and host galaxy, we convolve a pre-explosion HST WFC3 $F 160 W$ image with a Gaussian kernel to produce an image

\footnotetext{
${ }^{29}$ http://www.eso.org/observing/etc/skycalc/skycalc.htm
} 

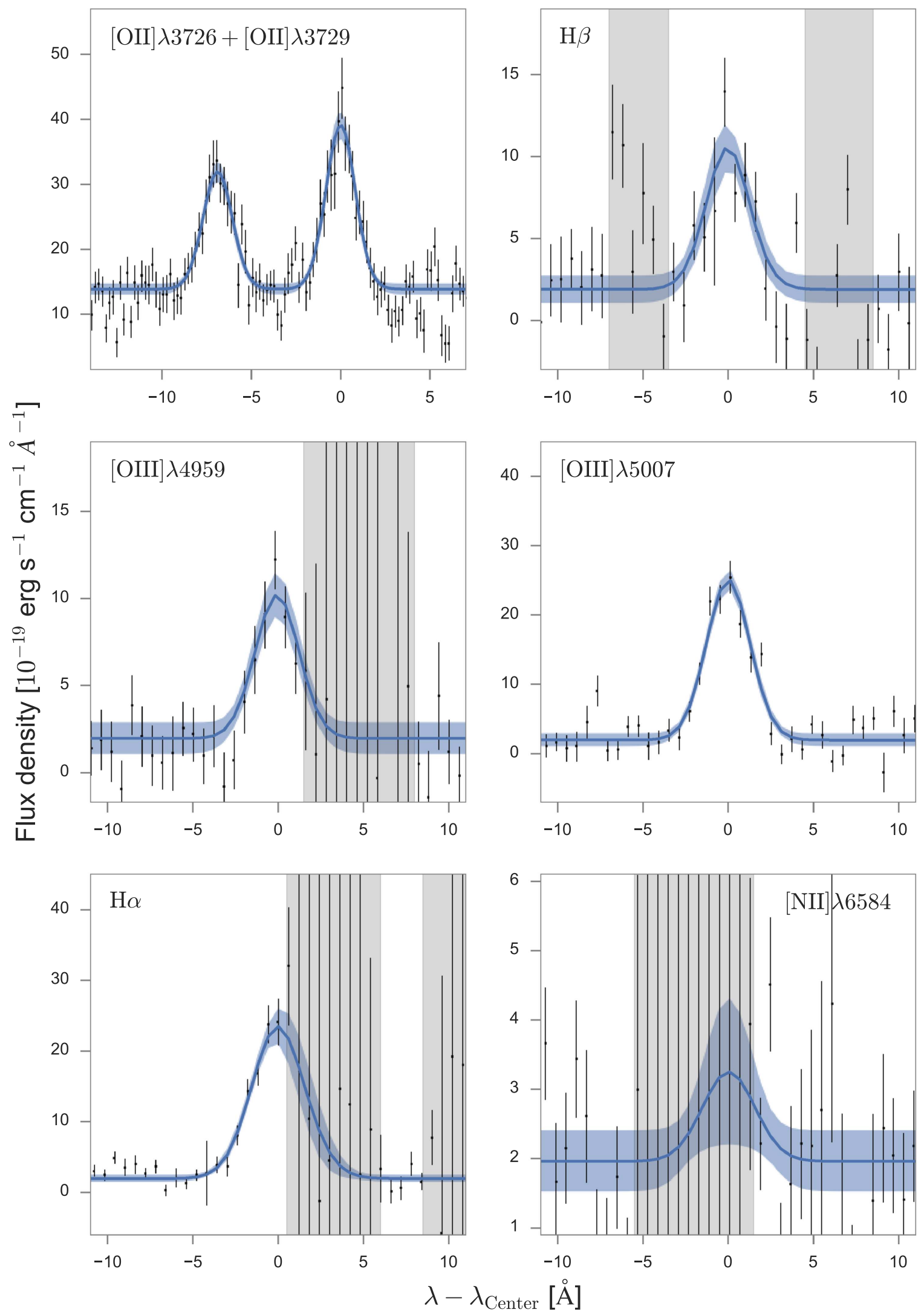

Figure 20. Profiles of narrow emission lines in X-shooter spectra at positions of SN images. Each fitted model (blue) consist of a Gaussian profile and a constant background. Regions having strong sky background from emission lines are shown by the gray background. Here we first find $\mu$ and $\sigma$ that yield the best fit to the [O III] $\lambda 5007$ emission line, and, during fits to other emission lines, we allow the line amplitude and local background to vary. The widths of the emission lines arising from forbidden transitions ([O III] $\lambda \lambda 4959,5007)$ and permitted transitions $(\mathrm{H} \alpha$ and $\mathrm{H} \beta)$ are comparable, which suggests that the narrow-line emission arises from $\mathrm{H}$ II regions instead of from the SN. 


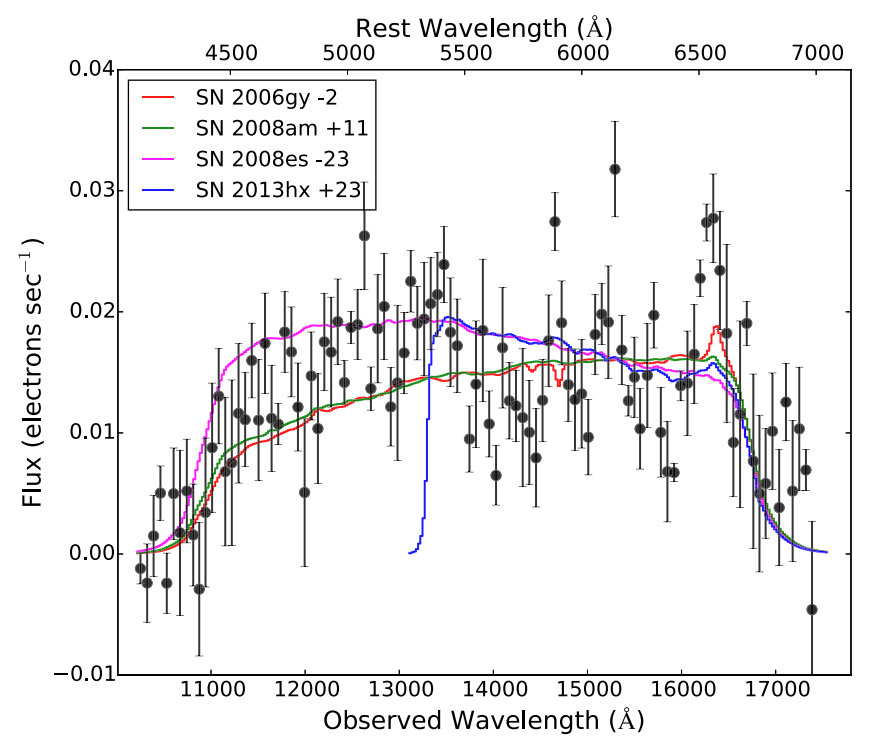

Figure 21. Comparison of grism spectra of SN Refsdal taken in both $111^{\circ}$ and $119^{\circ}$ orientations and binned in wavelength with the spectra of H-rich SLSNe. The $\mathrm{H} \alpha$ emission of SN Refsdal shows a much higher equivalent width than the spectra of the SLSNe exhibit. Each bin is $100 \AA$ in width, and plotted uncertainties are estimated through bootstrapping with replacement fluxes in the $20 \AA$ bins. The grism spectra contributing to this combined spectrum of SN Refsdal have phases of $-47 \pm 8$ days.

having the average FWHM expected during each X-shooter observation (see Table 2). From the PA and target positions, we next create pixel masks of the slit apertures. We use these masks and the convolved images to calculate the average expected SN and galaxy light admitted through the slit. We correct each spectrum for the slit losses appropriate for the SN point source.

We smooth each of the OBs separately and remove $5 \sigma$ outliers. The spectra are finally combined using a weighted average, and we propagate uncertainties.

\section{A.4. Estimating the Luminosity of the SN Ho Emission}

We measure the $\mathrm{H} \alpha$ strength by fitting a Gaussian to the emission after subtracting a continuum level. We estimate a median flux in the wavelength range $14500-15500 \AA$ for the grism spectrum. In the case of the $\mathrm{X}$-shooter data, the continuum estimate is the median of the flux in the range $15300-15600 \AA$ and $16500-17000 \AA$. The uncertainty is computed by repeating this procedure to the bootstrapresampled spectra.

To assemble the comparison plot in Figure 13, we renormalize the SN 1987A-like SNe and SN 2005cp comparison spectra to match published total magnitudes in the papers listed in Table 6. We next correct the spectra to remove Milky Way and host-galaxy reddening according to the values in Table 6 and shift the comparison spectra to the rest frame. The spectra of the low-redshift comparison $\mathrm{SNe}$ have high $\mathrm{S} / \mathrm{N}$, and we estimate the continuum visually from the region adjacent to their P-Cygni $\mathrm{H} \alpha$ profiles while avoiding the $\mathrm{Ba}$ II $\lambda 6142$ feature.

\section{A.4.1. Nebular Emission from Near the SN Position and Host Nuclear Region}

We identify the host-galaxy emission lines [O II] $\lambda 3727, \mathrm{H} \gamma$, $\mathrm{H} \beta,[\mathrm{O} \mathrm{III}] \lambda 4959,[\mathrm{O} \mathrm{III}] \lambda 5007, \mathrm{H} \alpha,[\mathrm{N} \mathrm{II}] \lambda 6584,[\mathrm{~S} \mathrm{II}]$ $\lambda 6716$, and [S II] $\lambda 6731$ with varying statistical significance. Narrow lines are detected across much of the spatial direction of the slit, which extends along both the host nucleus and position of $\mathrm{SN}$ images $\mathrm{S} 1$ and $\mathrm{S} 2$ for observations $\mathrm{OB} 1$ and OB2 and the position of SN images $\mathrm{S} 2$ and $\mathrm{S} 3$ for OB3. As an illustration, Figure 14 shows the detection of [O II] in OB2. The spatial dimension of the slit allows us to determine both the conditions local to the $\mathrm{SN}$ and within the nuclear region.

Parameters useful for the determination of the conditions of the emitting gas are the fluxes and widths of the strong nebular emission lines. Strong-line diagnostics using the measured line fluxes can then be used to infer the oxygen abundance of the emitting gas.

To estimate the uncertainty of the strong-line fluxes, we create bootstrapped spectra from the set of four separate spectra of images of the explosion site assembled from the OB1 and OB2 observations of SN images S1 and S2. Unlike the spectra taken during OB3, the spectra acquired in OB1 and OB2 show no evidence that emitting sources in the "off regions" were subtracted from the spectrum of the "on regions."

For each of the bootstrapped samples, the lines of [O II] $\lambda 3727, \mathrm{H} \beta,[\mathrm{O}$ III] $\lambda 4959,[\mathrm{O} \mathrm{III}] \lambda 5007, \mathrm{H} \alpha$, and [N II] $\lambda 6584$ are fit using weighted least-squares, where for the [O II] and [O III] doublets the widths are required to be the same, and the position and width of [N II] are determined from the fit to [O III] $\lambda 5007$ which is not contaminated by night-sky emission. We use the inverse variance as the weight. To estimate the uncertainty of the individual fits, we fit the spectrum assembled from each bootstrap combination 100 times after resampling within the uncertainty. We use the mean and the standard deviation of the resulting distribution to estimate the value and uncertainty of the line flux.

We do not detect [N II] in the spectrum extracted at the SN position, so we instead report an upper limit. To determine the value of the upper limit, we add an artificial line of increasing strength with a width matching that of the well-detected [O III] $\lambda 5007$ and repeat until we obtain a $3 \sigma$ detection.

For the host-galaxy nuclear region, we only have two extractions from $\mathrm{OB} 1$ and $\mathrm{OB} 2$, so we find a weighted combination of the two independent extractions. Because the strength of [O III] $\lambda 5007$ is weak at the position of the hostgalaxy nucleus, we use the width of [O II] instead of [O III] as a model for [N II] and let it vary within $3 \sigma$ of the best-fit values for [O II]. Using [O II] or [O III] to model the [N II] line profile has only a $\sim 5 \%$ effect on measured emission-line ratios. Figure 20 shows our fits to narrow nebular emission lines at the positions of the SN images.

\section{A.5. K-corrections}

For the purpose of computing the absolute magnitudes and colors of SN Refsdal at peak that are plotted in Figure 5, we adopt peak magnitudes of $F 105 W=24.90 \mathrm{mag} \mathrm{AB}$, $F 125 \mathrm{~W}=24.55 \mathrm{mag} \mathrm{AB}$ and $F 160 \mathrm{~W}=24.2 \mathrm{mag} \mathrm{AB}$ representative of the light curves of S1-S3. Since the WFC3 grism spectrum covers only $11000-17000 \AA(\sim 4400-6800 \AA$ in the rest frame) and the continuum is difficult to extract from the VLT spectrum, we use spectra of low-redshift SN 1987A-like SNe as well as SN 2005cp to compute $K$-corrections between the observer-frame WFC3 F105W, F125W, and F160W magnitudes and the rest-frame $B, V$, and $R$ filters. We define 
these color $K$-corrections as follows,

$$
\begin{aligned}
& B-V=m_{\mathrm{F} 105 \mathrm{~W}, \mathrm{syn}}^{\mathrm{AB}}-m_{\mathrm{F} 125 \mathrm{~W}, \mathrm{syn}}^{\mathrm{AB}}+K_{B-V}, \\
& V-R=m_{\mathrm{F} 125 \mathrm{~W}, \mathrm{syn}}^{\mathrm{AB}}-m_{\mathrm{F} 160 \mathrm{~W}, \mathrm{syn}}^{\mathrm{AB}}+K_{V-R},
\end{aligned}
$$

and

$$
V-r=m_{\mathrm{F} 125 \mathrm{~W}, \mathrm{syn}}^{\mathrm{AB}}-m_{\mathrm{F} 160 \mathrm{~W}, \mathrm{syn}}^{\mathrm{AB}}+K_{V-r} .
$$

Here the $K$-correction is defined following Kim et al. (1996),

$$
m_{y}=M_{x}+\mu(z)+K_{x y}
$$

where $m_{y}$ is the apparent magnitude in the $y$ band, $M_{x}$ is the absolute magnitude in the $x$ band, and $\mu(z)$ is the distance modulus.

We calculate $K$-corrections using spectra of SN $2006 \mathrm{~V}$ ( +6 days; $B-V=0.21 \mathrm{mag} ; V-r=-0.17 \mathrm{mag} ; V-R=$ $0.0 \mathrm{mag}), \quad \mathrm{SN} \quad 2006 \mathrm{au} \quad(-16$ days; $B-V=0.063 \mathrm{mag}$; $V-r=-0.18 \mathrm{mag} ; \quad V-R=-0.04 \mathrm{mag}), \quad \mathrm{SN} \quad 2005 \mathrm{cp}$ $(+14$ days; $B-V=0.21 \mathrm{mag} ; \quad V-r=0.0 \mathrm{mag} ; \quad V-R=$ $0.0 \mathrm{mag})$, and PTF 12gcx (+1 day; $B-V=0.306 \mathrm{mag}$; $V-r=-0.37 \mathrm{mag} ; V-R=-0.01 \mathrm{mag})$. All spectra were obtained within several weeks of maximum. In Figure 5, we adopt the representative values $K_{B-V}=0.2 \mathrm{mag}$, and $K_{V-R}=0.0 \mathrm{mag}$ to compute the rest-frame $B-V$ and $V-R$ colors of SN Refsdal near maximum.

In Figure 5, we show inferred absolute $B$ and $V$ rest-frame magnitudes of SN Refsdal, and, to compute these values, we next calculate the following $K$-corrections,

$$
K_{\mathrm{F} 105 \mathrm{~W} B}=2.5 \times \log _{10}(1+z)+m_{\mathrm{F} 105 \mathrm{~W}, \mathrm{syn}}^{\mathrm{AB}}-m_{\mathrm{B}, \mathrm{syn}}^{\mathrm{Vega}}
$$

and

$$
K_{\mathrm{F} 125 \mathrm{~W} V}=2.5 \times \log _{10}(1+z)+m_{\mathrm{F} 125 \mathrm{~W}, \mathrm{syn}}^{\mathrm{AB}}-m_{\mathrm{V}, \mathrm{syn}}^{\mathrm{Vega}} .
$$

We calculate these $K$-corrections for SN 2006V (+6 days; $\mathrm{F} 105 \mathrm{WB}=-0.86 \mathrm{mag} ; \mathrm{F} 125 \mathrm{WV}=-0.80 \mathrm{mag}), \mathrm{SN} 2006 \mathrm{au}$ $(-16$ days; F105WB $=-0.85 \mathrm{mag} ; \mathrm{F} 125 \mathrm{WV}=-0.788 \mathrm{mag})$, $\mathrm{SN} 2005 \mathrm{cp}(+14$ days; $\mathrm{F} 105 \mathrm{WB}=-1.01 \mathrm{mag} ; \mathrm{F} 125 \mathrm{WV}=$ $-0.80 \mathrm{mag})$, and PTF $12 \mathrm{gcx}(+1$ day $\mathrm{F} 105 \mathrm{WB}=-0.91 \mathrm{mag}$; $\mathrm{F} 125 \mathrm{WV}=-0.69 \mathrm{mag})$. We adopt the representative values $K_{\mathrm{F} 105 \mathrm{~W} B}=-0.9 \mathrm{mag}$, and $K_{\mathrm{F} 125 \mathrm{WV}}=-0.8 \mathrm{mag}$ to compute the absolute $B$ and $V$ magnitudes of SN Refsdal near maximum plotted in Figure 5.

In Figure 4, we have instead transformed the $B-V$ and $V-R$ colors of the low-redshift $\mathrm{SN}$ to $F 105 W-F 125 W$ and $F 125 W-F 160 \mathrm{~W}$ at redshift $z=1.49$. While Figure 5 presents a comparison of colors and absolute magnitudes at maximum light, Figure 4 shows the evolution of colors with SN phase. Since $K$-corrections are needed across a wide range of phase, we therefore use the spectra of the extremely well-sampled SN 1987A as a model. Using spectra of SN 1987A instead of the average of the SN samples used to compute $K$-corrections for Figure 5 above leads to a small, $\sim 0.2 \mathrm{mag}$, difference in the $K$-correction at maximum light.

\section{A.6. Comparison with the Spectra} of H-rich Superluminous Supernovae

In Figure 21, we show a comparison between the WFC3 G141 grism spectrum of SN Refsdal and the spectra of four SLSNe. The $\mathrm{H} \alpha$ emission from SN Refsdal has a significantly higher equivalent width (EW) than those of the $\mathrm{H} \alpha$ emission features of the SLSNe. The spectra of the superluminous comparison objects are drawn from the literature (SN 2006gy: Smith et al. 2007; SN 2008am: Chatzopoulos et al. 2011; SN 2008es: Gezari et al. 2009; SN 2013hx: Inserra et al. 2016).

\section{REFERENCES}

Akaike, H. 1974, ITAC, 19, 716

Anderson, J. P., Covarrubias, R. A., James, P. A., Hamuy, M., \& Habergham, S. M. 2010, MNRAS, 407, 2660

Applegate, D. E., von der Linden, A., Kelly, P. L., et al. 2014, MNRAS, 439, 48

Arnett, W. D. 1979, ApJL, 230, L37

Arnett, W. D., Bahcall, J. N., Kirshner, R. P., \& Woosley, S. E. 1989, ARA\&A, 27, 629

Baldwin, J. A., Phillips, M. M., \& Terlevich, R. 1981, PASP, 93, 5

Barbon, R., Ciatti, F., \& Rosino, L. 1979, A\&A, 72, 287

Blinnikov, S., Lundqvist, P., Bartunov, O., Nomoto, K., \& Iwamoto, K. 2000 ApJ, 532, 1132

Brammer, G. B., Sánchez-Janssen, R., Labbé, I., et al. 2012a, ApJL, 758, L17

Brammer, G. B., van Dokkum, P. G., Franx, M., et al. 2012b, ApJS, 200, 13

Chatzopoulos, E., Wheeler, J. C., Vinko, J., et al. 2011, ApJ, 729, 143

Chugai, N. N. 2001, MNRAS, 326, 1448

Cooper, M. C., Newman, J. A., Davis, M., Finkbeiner, D. P., \& Gerke, B. F. 2012, spec2d: DEEP2 DEIMOS Spectral Pipeline, Astrophysics Source Code Library, ascl:1203.003

Crotts, A. P. S., \& Heathcote, S. R. 2000, ApJ, 528, 426

Diego, J. M., Broadhurst, T., Chen, C., et al. 2015, MNRAS, 456, 356

Doggett, J. B., \& Branch, D. 1985, AJ, 90, 2303

Domínguez, A., Siana, B., Henry, A. L., et al. 2013, ApJ, 763, 145

Ebeling, H., Edge, A. C., \& Henry, J. P. 2001, ApJ, 553, 668

Faber, S. M., Phillips, A. C., Kibrick, R. I., et al. 2003, Proc. SPIE, 4841, 1657 Filippenko, A. V. 1982, PASP, 94, 715

Filippenko, A. V. 1997, ARA\&A, 35, 309

Freudling, W., Romaniello, M., Bramich, D. M., et al. 2013, A\&A, 559, A96 Gezari, S., Halpern, J. P., Grupe, D., et al. 2009, ApJ, 690, 1313

Gilmozzi, R., Cassatella, A., Clavel, J., et al. 1987, Natur, 328, 318

Gonzaga, S. E., et al. 2012, The DrizzlePac Handbook, HST Data Handbook (New York: STSci)

Grillo, C., Karman, W., Suyu, S. H., et al. 2016, ApJ, 822, 75

Hamuy, M., \& Suntzeff, N. B. 1990, AJ, 99, 1146

Hamuy, M., Suntzeff, N. B., Heathcote, S. R., et al. 1994, PASP, 106, 566

Howell, D. A., Sullivan, M., Perrett, K., et al. 2005, ApJ, 634, 1190

Inserra, C., Smartt, S. J., Gall, E. E. E., et al. 2016, ApJ, submitted (arXiv:1604.01226)

Jauzac, M., Richard, J., Limousin, M., et al. 2016, MNRAS, 457, 2029

Karman, W., Grillo, C., Balestra, I., et al. 2016, A\&A, 585, A27

Kass, R. E., \& Raftery, A. E. 1995, J. Amer. Amer. Stat. Assn., 90, 773

Kawamata, R., Oguri, M., Ishigaki, M., Shimasaku, K., \& Ouchi, M. 2015, arXiv: 1510.06400

Kelly, P. L., \& Kirshner, R. P. 2012, ApJ, 759, 107

Kelly, P. L., Kirshner, R. P., \& Pahre, M. 2008, ApJ, 687, 1201

Kelly, P. L., Rodney, S. A., Treu, T., et al. 2015a, arXiv:1512.04654

Kelly, P. L., Rodney, S. A., Treu, T., et al. 2015b, Sci, 347, 1123

Kelly, P. L., von der Linden, A., Applegate, D. E., et al. 2014, MNRAS, 439, 28

Kiewe, M., Gal-Yam, A., Arcavi, I., et al. 2012, ApJ, 744, 10

Kim, A., Goobar, A., \& Perlmutter, S. 1996, PASP, 108, 190

Kleiser, I. K. W., Poznanski, D., Kasen, D., et al. 2011, MNRAS, 415, 372

Larkin, J., Barczys, M., Krabbe, A., et al. 2006, Proc. SPIE, 6269, 62691

McLean, I. S., Steidel, C. C., Epps, H., et al. 2010, Proc. SPIE, 7735, 77351

McLean, I. S., Steidel, C. C., Epps, H. W., et al. 2012, Proc. SPIE, 8446, 84460

Meaburn, J., Bryce, M., \& Holloway, A. J. 1995, A\&A, 299, L1

Miller, A. A., Chornock, R., Perley, D. A., et al. 2009, ApJ, 690, 1303

Modigliani, A., Goldoni, P., Royer, F., et al. 2010, Proc. SPIE, 7737, 28

Momcheva, I. G., Brammer, G. B., van Dokkum, P. G., et al. 2016, ApJS, 225,27

Mukherjee, S., Feigelson, E. D., Jogesh Babu, G., et al. 1998, ApJ, 508, 314 Newman, J. A., Cooper, M. C., Davis, M., et al. 2013, ApJS, 208, 5

Oguri, M. 2015, MNRAS, 449, L86

Pagel, B. E. J., Edmunds, M. G., Blackwell, D. E., Chun, M. S., \& Smith, G. 1979, MNRAS, 189, 95

Pastorello, A., Baron, E., Branch, D., et al. 2005, MNRAS, 360, 950

Pastorello, A., Pumo, M. L., Navasardyan, H., et al. 2012, A\&A, 537, A141

Pastorello, A., Zampieri, L., Turatto, M., et al. 2004, MNRAS, 347, 74 
Pettini, M., \& Pagel, B. E. J. 2004, MNRAS, 348, L59

Phillips, M. M., Hamuy, M., Heathcote, S. R., Suntzeff, N. B., \& Kirhakos, S. 1990, AJ, 99, 1133

Phillips, M. M., Heathcote, S. R., Hamuy, M., \& Navarrete, M. 1988, AJ, 95, 1087

Quimby, R. M., Yuan, F., Akerlof, C., \& Wheeler, J. C. 2013, MNRAS, 431, 912

Reddy, N. A., Kriek, M., Shapley, A. E., et al. 2015, ApJ, 806, 259

Refsdal, S. 1964, MNRAS, 128, 307

Rodney, S. A., Strolger, L.-G., Kelly, P. L., et al. 2016, ApJ, 820, 50

Schlafly, E. F., \& Finkbeiner, D. P. 2011, ApJ, 737, 103

Schmidt, K. B., Treu, T., Brammer, G. B., et al. 2014, ApJL, 782, L36

Sharon, K., \& Johnson, T. L. 2015, ApJL, 800, L26

Silverman, J. M., Foley, R. J., Filippenko, A. V., et al. 2012, MNRAS, 425, 1789

Smartt, S. J., Eldridge, J. J., Crockett, R. M., \& Maund, J. R. 2009, MNRAS, 395, 1409

Smith, G. P., Ebeling, H., Limousin, M., et al. 2009, ApJL, 707, L163

Smith, N., Chornock, R., Silverman, J. M., Filippenko, A. V., \& Foley, R. J. 2010, ApJ, 709, 856

Smith, N., Li, W., Foley, R. J., et al. 2007, ApJ, 666, 1116

Smith, N., Mauerhan, J. C., Cenko, S. B., et al. 2015, MNRAS, 449, 1876

Smith, N., Mauerhan, J. C., \& Prieto, J. L. 2014, MNRAS, 438, 1191
Sonneborn, G., Altner, B., \& Kirshner, R. P. 1987, ApJL, 323, L35

Staveley-Smith, L., Kim, S., Calabretta, M. R., Haynes, R. F., \& Kesteven, M. J. 2003, MNRAS, 339, 87

Sugiura, N. 1978, Commun. Stat. A-Theor, 13

Taddia, F., Sollerman, J., Fremling, C., et al. 2016, A\&A, 588, A5

Taddia, F., Sollerman, J., Razza, A., et al. 2013, A\&A, 558, A143

Taddia, F., Stritzinger, M. D., Sollerman, J., et al. 2012, A\&A, 537, A140

Thomas, D., Steele, O., Maraston, C., et al. 2013, MNRAS, 431, 1383

Tonry, J., \& Davis, M. 1979, AJ, 84, 1511

Treu, T., Brammer, G., Diego, J. M., et al. 2016, ApJ, 817, 60

Treu, T., \& Ellis, R. S. 2015, ConPh, 56, 17

Treu, T., Schmidt, K. B., Brammer, G. B., et al. 2015, ApJ, 812, 114

Vernet, J., Dekker, H., D’Odorico, S., et al. 2011, A\&A, 536, A105

Vernet, J., Kerber, F., Mainieri, V., et al. 2010, HiA, 15, 535

von der Linden, A., Allen, M. T., Applegate, D. E., et al. 2014, MNRAS, 439, 2

Welty, D. E., Xue, R., \& Wong, T. 2012, ApJ, 745, 173

Yaron, O., \& Gal-Yam, A. 2012, PASP, 124, 668

Yuan, T., Kobayashi, C., \& Kewley, L. J. 2015, ApJL, 804, L14

Yuan, T.-T., Kewley, L. J., Swinbank, A. M., Richard, J., \& Livermore, R. C. 2011, ApJL, 732, L14

Zitrin, A., \& Broadhurst, T. 2009, ApJL, 703, L132 